TRANSACTIONS OF THE

AMERICAN MATHEMATICAL SOCIETY

Volume 361, Number 3, March 2009, Pages 1371-1395

S 0002-9947(08)04636-9

Article electronically published on October 17, 2008

\title{
MONOMIAL AND TORIC IDEALS ASSOCIATED TO FERRERS GRAPHS
}

\author{
ALBERTO CORSO AND UWE NAGEL
}

\begin{abstract}
Each partition $\lambda=\left(\lambda_{1}, \lambda_{2}, \ldots, \lambda_{n}\right)$ determines a so-called Ferrers tableau or, equivalently, a Ferrers bipartite graph. Its edge ideal, dubbed a Ferrers ideal, is a squarefree monomial ideal that is generated by quadrics. We show that such an ideal has a 2-linear minimal free resolution; i.e. it defines a small subscheme. In fact, we prove that this property characterizes Ferrers graphs among bipartite graphs. Furthermore, using a method of Bayer and Sturmfels, we provide an explicit description of the maps in its minimal free resolution. This is obtained by associating a suitable polyhedral cell complex to the ideal/graph. Along the way, we also determine the irredundant primary decomposition of any Ferrers ideal. We conclude our analysis by studying several features of toric rings of Ferrers graphs. In particular we recover/establish formulæ for the Hilbert series, the Castelnuovo-Mumford regularity, and the multiplicity of these rings. While most of the previous works in this highly investigated area of research involve path counting arguments, we offer here a new and self-contained approach based on results from Gorenstein liaison theory.
\end{abstract}

\section{INTRODUCTION}

A Ferrers graph is a bipartite graph on two distinct vertex sets $\mathbf{X}=\left\{x_{1}, \ldots, x_{n}\right\}$ and $\mathbf{Y}=\left\{y_{1}, \ldots, y_{m}\right\}$ such that if $\left(x_{i}, y_{j}\right)$ is an edge of $G$, then so is $\left(x_{p}, y_{q}\right)$ for $1 \leq p \leq i$ and $1 \leq q \leq j$. In addition, $\left(x_{1}, y_{m}\right)$ and $\left(x_{n}, y_{1}\right)$ are required to be edges of $G$. For any Ferrers graph $G$ there is an associated sequence of non-negative integers $\lambda=\left(\lambda_{1}, \lambda_{2}, \ldots, \lambda_{n}\right)$, where $\lambda_{i}$ is the degree of the vertex $x_{i}$. Notice that the defining properties of a Ferrers graph imply that $\lambda_{1}=m \geq \lambda_{2} \geq \cdots \geq \lambda_{n} \geq 1$; thus $\lambda$ is a partition. Alternatively, we can associate to a Ferrers graph a diagram $\mathbf{T}_{\lambda}$, dubbed a Ferrers tableau, consisting of an array of $n$ rows of cells with $\lambda_{i}$ adjacent cells, left justified, in the $i$-th row.

Ferrers graphs/tableaux have a prominent place in the literature as they have been studied in relation to chromatic polynomials [2, 18], Schubert varieties [16, 15], hypergeometric series [29, permutation statistics [9, 18, quantum mechanical operators [50, and inverse rook problems [23, 16, 15, 42]. More generally, algebraic and combinatorial aspects of bipartite graphs have been studied in depth (see, e.g., [46, 30, and the comprehensive monograph [51]). In this paper, which will be followed by [13, we are interested in the algebraic properties of the edge ideal

Received by the editors January 22, 2007.

2000 Mathematics Subject Classification. Primary 05A15, 13D02, 13D40, 14M25; Secondary 05C $75,13 \mathrm{C} 40,13 \mathrm{H} 10,14 \mathrm{M} 12,52 \mathrm{~B} 05$.

The second author gratefully acknowledges partial support from the NSA under grant H9823007-1-0065.

(C)2008 American Mathematical Society
Reverts to public domain 28 years from publication 1371 
$I=I(G)$ and the toric ring $K[G]$ associated to a Ferrers graph $G$. The edge ideal is the monomial ideal of the polynomial ring $R=K\left[x_{1}, \ldots, x_{n}, y_{1}, \ldots, y_{m}\right]$ over the field $K$ that is generated by the monomials of the form $x_{i} y_{j}$, whenever the pair $\left(x_{i}, y_{j}\right)$ is an edge of $G . K[G]$ is instead the monomial subalgebra generated by the elements $x_{i} y_{j}$. An example is illustrated in Figure 1.

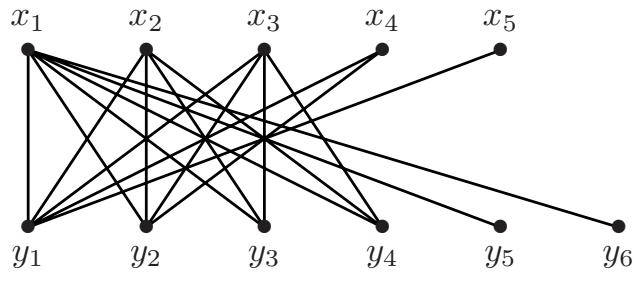

Ferrers graph

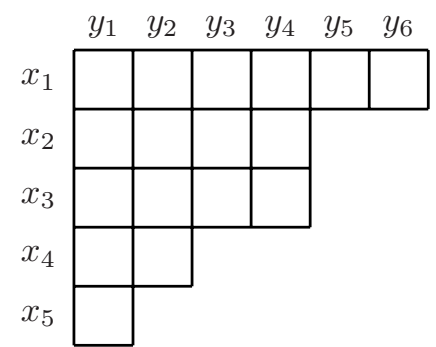

Ferrers tableau with partition $\lambda=(6,4,4,2,1)$

$$
\begin{gathered}
I=\left(x_{1} y_{1}, x_{1} y_{2}, x_{1} y_{3}, x_{1} y_{4}, x_{1} y_{5}, x_{1} y_{6}, x_{2} y_{1}, x_{2} y_{2}, x_{2} y_{3}, x_{2} y_{4},\right. \\
\left.x_{3} y_{1}, x_{3} y_{2}, x_{3} y_{3}, x_{3} y_{4}, x_{4} y_{1}, x_{4} y_{2}, x_{5} y_{1}\right)
\end{gathered}
$$

Figure 1. Ferrers graph, tableau and ideal

Throughout this article $\lambda=\left(\lambda_{1}, \ldots, \lambda_{n}\right)$ will always denote a fixed partition associated to a Ferrers graph $G_{\lambda}$ with corresponding Ferrers ideal $I_{\lambda}$. In Section 2 we describe several fine numerical invariants attached to the ideal $I_{\lambda}$. In Theorem 2.1 we show that each Ferrers ideal defines a small subscheme in the sense of Eisenbud, Green, Hulek, and Popescu 20; i.e. the free resolution of $I_{\lambda}$ is 2-linear (see also Remark 3.31). More precisely, we give an explicit — but at the same time surprisingly simple - formula for the Betti numbers of the ideal $I_{\lambda}$; namely, we show that

$$
\beta_{i}\left(R / I_{\lambda}\right)=\left(\begin{array}{c}
\lambda_{1} \\
i
\end{array}\right)+\left(\begin{array}{c}
\lambda_{2}+1 \\
i
\end{array}\right)+\left(\begin{array}{c}
\lambda_{3}+2 \\
i
\end{array}\right)+\ldots+\left(\begin{array}{c}
\lambda_{n}+n-1 \\
i
\end{array}\right)-\left(\begin{array}{c}
n \\
i+1
\end{array}\right)
$$

for $1 \leq i \leq \max \left\{\lambda_{i}+i-1\right\}$. Furthermore, the Hilbert series is

$$
\sum_{k \geq 0} \operatorname{dim}_{K}\left[R / I_{\lambda}\right]_{k} \cdot t^{k}=\frac{1}{(1-t)^{m}}+\frac{t}{(1-t)^{m+n+1}} \cdot \sum_{j=1}^{n}(1-t)^{\lambda_{j}+j} .
$$

Notice that the formula for the Betti numbers involves a minus sign. This is quite an unusual phenomenon for Betti numbers, as they tend, in general, to have an enumerative interpretation. In order to determine the Betti numbers it is essential to find a (not necessarily irredundant) primary decomposition of $I_{\lambda}$. We refine this decomposition into an irredundant one in Corollary 2.6. where we observe, in particular, that the number of prime components is related to the outer corners of the Ferrers tableau. For instance, in the case of the ideal $I_{\lambda}$ described in Figure 1 we have that it is the intersection of $5(=4$ outer corners +1$)$ components:

$$
\begin{aligned}
I_{\lambda}=\left(y_{1}, \ldots, y_{6}\right) & \cap\left(x_{1}, y_{1}, y_{2}, y_{3}, y_{4}\right) \\
& \cap\left(x_{1}, x_{2}, x_{3}, y_{1}, y_{2}\right) \cap\left(x_{1}, x_{2}, x_{3}, x_{4}, y_{1}\right) \cap\left(x_{1}, \ldots, x_{5}\right) .
\end{aligned}
$$


We conclude Section 2 by identifying, in terms of the shape of the tableau, the unmixed (Corollary 2.7) and Cohen-Macaulay (Corollary 2.8) members in the family of Ferrers ideals. The latter result also follows from recent work of Herzog and Hibi 30.

There are relatively few general classes of ideals for which an explicit minimal free resolution is known. The most noteworthy such families include the Koszul complex, the Eagon-Northcott complex [17, and the resolution of generic monomial ideals [3] (see also 44). In Section 3 we analyze even further the minimal free resolution of a Ferrers ideal $I_{\lambda}$ and obtain a surprisingly elegant description of the differentials in the resolution in Theorem 3.2 In some sense, this is a prototypical result as it provides the minimal free resolution of several classes of ideals obtained from Ferrers ideals by appropriate specializations of the variables (see [13] for further details). Our description of the free resolution of a Ferrers ideal relies on the theory of cellular resolutions as developed by Bayer and Sturmfels in [3] (see also 41]). More precisely, let $\Delta_{n-1} \times \Delta_{m-1}$ denote the product of two simplices of dimensions $n-1$ and $m-1$, respectively. Given a Ferrers ideal $I_{\lambda}$, we associate to it the polyhedral cell complex $X_{\lambda}$ consisting of the faces of $\Delta_{n-1} \times \Delta_{m-1}$ whose vertices are labeled by generators of $I_{\lambda}$ (see Definition 3.1). By the theory of Bayer and Sturmfels, $X_{\lambda}$ determines a complex of free modules. Using an inductive argument we show in Theorem 3.2 that this complex is in fact the multigraded minimal free resolution of the ideal $I_{\lambda}$. While leaving the details to the main body of the paper, we illustrate the situation in the case of the partition $\lambda=(4,3,2,1)$, which is the largest we can draw. In this case the polyhedral cell complex $X_{\lambda}$ can actually be identified with the subdivision of the simplex $\Delta_{3}$ pictured below (see [13] for additional details):

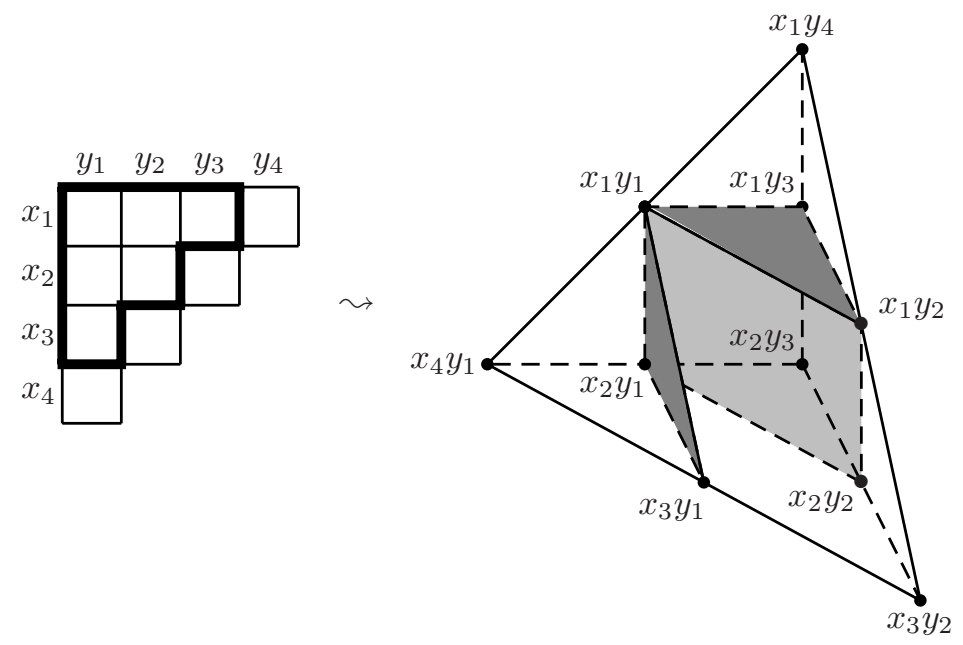

FiguRE 2. Ferrers tableau and associated polyhedral cell complex

In particular, we observe that $X_{\lambda}$ has four 3-dimensional cells: two of them are isomorphic to $\Delta_{3}$ whereas the remaining two are isomorphic to either $\Delta_{1} \times \Delta_{2}$ or 
$\Delta_{2} \times \Delta_{1}$. A grey shading in the picture above also indicates how the polyhedral cell complex corresponding to the partition $(3,2,1)$ sits inside $X_{\lambda}$.

In Section 4 we prove the converse of Theorem 2.1 Namely, we show that any edge ideal of a bipartite graph with a 2-linear resolution necessarily arises from a Ferrers graph (see Theorem 4.2). One of the ingredients of the proof is a wellknown characterization of edge ideals of graphs with a 2-linear resolution in terms of complementary graphs, due to Fröberg [21] (see also [19]).

The starting point of Section 5 is the observation that the toric ring of a Ferrers graph can be identified with a special ladder determinantal ring. We then proceed to recover/establish formulæ for the Hilbert series and other invariants associated with these rings. We remark that this is a highly investigated part of mathematics that has been the subject of the work of many researchers. Among the extensive, impressive and relevant literature we single out 1, 8, 10, 11, 12, 26, 32, 34, 35, 36, 37, 38, 43, 45, 52. While most of these works involve - to a different extent - path counting arguments, we offer here a new and self-contained approach that yields easy proofs of explicit formulæ for the Hilbert series, the Castelnuovo-Mumford regularity, and the multiplicity of the toric rings of Ferrers graphs. This method, which is based on results from Gorenstein liaison theory (see 39] for a comprehensive introduction), has been pioneered in 33, where it was proved that every standard determinantal ideal is glicci; i.e. it is in the Gorenstein liaison class of a complete intersection (see also [40]). Recently, Gorla 24 has considerably refined these arguments to show that all ladder determinantal ideals are glicci. This result can be used to establish first a simple recursive formula for the Hilbert series, which we then turn into an explicit formula that involves only positive summands. We end by saying that the numerical data we explicitly determine also allow us to compute the reduction number of Ferrers ideals, which is a key ingredient in determining the structure of the core of ideals. Indeed, some classes of monomial ideals possess a surprising interpretation for their core (see 44]). Preliminary investigations also show that the core of a Ferrers ideal has a remarkable structure related to the shape of the corresponding Ferrers tableau.

\section{BetTi numbers AND PRIMARY DECOMPOSITIONS OF FERRERS IDEALS}

The main result of this section, Theorem 2.1. provides a (not necessarily irredundant) primary decomposition of a Ferrers ideal $I_{\lambda}$ as well as the Betti numbers of its minimal free resolution. A particularly relevant situation is given by a maximal bipartite graph, in which case the Ferrers tableau has a rectangular shape of size $n \times m$ and $I_{\lambda}=\left(x_{1}, \ldots, x_{n}\right)\left(y_{1}, \ldots, y_{m}\right)$; using a variety of techniques, including determinantal ideals, residual intersections and Gröbner basis techniques, 14] and 6] describe additional features of this and other related subideals in connection with the so-called Dedekind-Mertens Lemma. A hook-shaped tableau is the other extremal case; in this situation, $I_{\lambda}=x_{1}\left(y_{1}, \ldots, y_{m}\right)+y_{1}\left(x_{1}, \ldots, x_{n}\right)$.

For the sake of simplicity we will denote the partition associated to a Ferrers graph by $\lambda=\left(\lambda_{1}, \lambda_{2}, \ldots, \lambda_{s}, 1, \ldots, 1\right)$ with $\lambda_{1} \geq \lambda_{2} \geq \cdots \geq \lambda_{s} \geq 2$. Furthermore, we denote the dual partition by $\lambda^{*}=\left(\lambda_{1}^{*}, \lambda_{2}^{*}, \ldots, \lambda_{m}^{*}\right)$, where $\lambda_{j}^{*}$ is the degree of the vertex $y_{j}$. Observe that $\lambda_{1}=m, \lambda_{1}^{*}=n$, and $1 \leq s=\lambda_{2}^{*} \leq n$. We also recall that the Hilbert series of the graded $K$-algebra $R / I_{\lambda}$ is

$$
P\left(R / I_{\lambda}, t\right):=\sum_{k \geq 0} h_{R / I_{\lambda}}(k) \cdot t^{k}:=\sum_{k \geq 0} \operatorname{dim}_{K}\left[R / I_{\lambda}\right]_{k} \cdot t^{k},
$$


where $h_{R / I_{\lambda}}$ is the Hilbert function of $R / I_{\lambda}$. It is well known that this series is a rational function.

Theorem 2.1. Let $G$ be a Ferrers graph with associated partition $\lambda=\left(\lambda_{1}, \lambda_{2}, \ldots\right.$, $\left.\lambda_{s}, 1, \ldots, 1\right)$ and let $I_{\lambda}$ be the edge ideal in $K\left[x_{1}, \ldots, x_{n}, y_{1}, \ldots, y_{m}\right]$ associated to $G$. Then a (not necessarily irredundant) primary decomposition of $I_{\lambda}$ is

$$
\begin{aligned}
& \left(y_{1}, \ldots, y_{\lambda_{1}}\right) \cap\left(x_{1}, y_{1}, \ldots, y_{\lambda_{2}}\right) \cap\left(x_{1}, x_{2}, y_{1}, \ldots, y_{\lambda_{3}}\right) \cap \ldots \\
& \cap\left(x_{1}, \ldots, x_{s-1}, y_{1}, \ldots, y_{\lambda_{s}}\right) \cap\left(x_{1}, \ldots, x_{s}, y_{1}\right) \cap\left(x_{1}, \ldots, x_{n}\right),
\end{aligned}
$$

and the minimal $\mathbb{Z}$-graded free resolution of $R / I_{\lambda}$ is 2-linear with $i$-th Betti number given by

$$
\beta_{i}\left(R / I_{\lambda}\right)=\left(\begin{array}{c}
\lambda_{1} \\
i
\end{array}\right)+\left(\begin{array}{c}
\lambda_{2}+1 \\
i
\end{array}\right)+\left(\begin{array}{c}
\lambda_{3}+2 \\
i
\end{array}\right)+\ldots+\left(\begin{array}{c}
\lambda_{n}+n-1 \\
i
\end{array}\right)-\left(\begin{array}{c}
n \\
i+1
\end{array}\right)
$$

for $1 \leq i \leq \max _{j}\left\{\lambda_{j}+j-1\right\}$. Furthermore, the Hilbert series is

$$
P\left(R / I_{\lambda}, t\right)=\frac{1}{(1-t)^{m}}+\frac{t}{(1-t)^{m+n+1}} \cdot \sum_{j=1}^{n}(1-t)^{\lambda_{j}+j} .
$$

Proof. We proceed by induction on $\lambda_{1}+\cdots+\lambda_{n}>0$. If this sum is one, then $I_{\lambda}=\left(x_{1} y_{1}\right)$ and all the claims are easily verified. Otherwise, we use induction on $n$. If $n=1$, then $\lambda=\left(\lambda_{1}\right)=(m), s=1$ and $I_{\lambda}=x_{1}\left(y_{1}, \ldots, y_{m}\right)=\left(x_{1}\right) \cap$ $\left(y_{1}, \ldots, y_{m}\right)=\left(y_{1}, \ldots, y_{m}\right) \cap\left(x_{1}, y_{1}\right) \cap\left(x_{1}\right)$. Moreover, the resolution of $I$ is given by (a shifted) Koszul complex on $m$ generators. Hence the $i$-th Betti number is

$$
\beta_{i}\left(R / I_{\lambda}\right)=\left(\begin{array}{c}
m \\
i
\end{array}\right)=\left(\begin{array}{c}
m \\
i
\end{array}\right)-\left(\begin{array}{c}
1 \\
i+1
\end{array}\right),
$$

as the latter term is zero. Furthermore, the Hilbert series is

$$
P\left(R / I_{\lambda}, t\right)=\frac{1}{(1-t)^{m}}+\frac{t}{(1-t)},
$$

as claimed.

Suppose now $n \geq 2$. We distinguish two main cases: $\lambda_{n}=1$ and $\lambda_{n} \geq 2$. We first deal with the case $\lambda_{n}=1$. In addition to the partition $\lambda$ we consider the partition $\lambda^{\prime}=\left(\lambda_{1}, \ldots, \lambda_{n-1}\right)$. Notice that the index $s$ is the same for both $\lambda$ and $\lambda^{\prime}$. By the induction hypothesis we have that a primary decomposition of $I_{\lambda^{\prime}}$ is

$$
\begin{aligned}
\left(y_{1}, \ldots, y_{\lambda_{1}}\right) & \cap\left(x_{1}, y_{1}, \ldots, y_{\lambda_{2}}\right) \cap\left(x_{1}, x_{2}, y_{1}, \ldots, y_{\lambda_{3}}\right) \cap \ldots \\
& \cap\left(x_{1}, \ldots, x_{s-1}, y_{1}, \ldots, y_{\lambda_{s}}\right) \cap\left(x_{1}, \ldots, x_{s}, y_{1}\right) \cap\left(x_{1}, \ldots, x_{n-1}\right) .
\end{aligned}
$$

Let $J$ denote the intersection of all the components in the above primary decomposition that contain $x_{n} y_{1}$. Thus, $I_{\lambda^{\prime}}=J \cap\left(x_{1}, \ldots, x_{n-1}\right)$. Now observe that $I_{\lambda}=I_{\lambda^{\prime}}+\left(x_{n} y_{1}\right)$; thus using the above primary decomposition for $I_{\lambda^{\prime}}$, we get

$$
\begin{aligned}
I_{\lambda} & =J \cap\left(x_{1}, \ldots, x_{n-1}, x_{n} y_{1}\right) \\
& =J \cap\left(x_{1}, \ldots, x_{n-1}, y_{1}\right) \cap\left(x_{1}, \ldots, x_{n-1}, x_{n}\right) \\
& =J \cap\left(x_{1}, \ldots, x_{n}\right),
\end{aligned}
$$

which is, after some inspection, exactly the asserted primary decomposition of $I_{\lambda}$. 
We now turn to the Betti numbers of $I_{\lambda}$. From the given primary decomposition, it follows that $I_{\lambda^{\prime}}: x_{n} y_{1}=\left(x_{1}, \ldots, x_{n-1}\right)$. Hence we have the following short exact sequence:

$$
0 \rightarrow R /\left(x_{1}, \ldots, x_{n-1}\right)[-2] \stackrel{\cdot x_{n} y_{1}}{\longrightarrow} R / I_{\lambda^{\prime}} \longrightarrow R / I_{\lambda} \rightarrow 0 .
$$

Using a mapping cone construction we obtain that

$$
\beta_{i}\left(R / I_{\lambda}\right)=\beta_{i}\left(R / I_{\lambda^{\prime}}\right)+\beta_{i-1}\left(R /\left(x_{1}, \ldots, x_{n-1}\right)\right)=\beta_{i}\left(R / I_{\lambda^{\prime}}\right)+\left(\begin{array}{c}
n-1 \\
i-1
\end{array}\right) .
$$

Hence, using our inductive assumption, we have that $\beta_{i}\left(R / I_{\lambda}\right)$ is given by

$$
\left(\begin{array}{c}
\lambda_{1} \\
i
\end{array}\right)+\left(\begin{array}{c}
\lambda_{2}+1 \\
i
\end{array}\right)+\left(\begin{array}{c}
\lambda_{3}+2 \\
i
\end{array}\right)+\ldots+\left(\begin{array}{c}
\lambda_{n-1}+n-2 \\
i
\end{array}\right)-\left(\begin{array}{c}
n-1 \\
i+1
\end{array}\right)+\left(\begin{array}{c}
n-1 \\
i-1
\end{array}\right) .
$$

However, one can easily check the identity $\left(\begin{array}{c}n-1 \\ i-1\end{array}\right)-\left(\begin{array}{c}n-1 \\ i+1\end{array}\right)=\left(\begin{array}{c}n \\ i\end{array}\right)-\left(\begin{array}{c}n \\ i+1\end{array}\right)$, which provides the expected form of $\beta_{i}\left(R / I_{\lambda}\right)$.

Moreover, since the resolution of $R /\left(x_{1}, \ldots, x_{n-1}\right)$ is linear, the mapping cone procedure applied to sequence (2.1) also shows that the resolution of $R / I_{\lambda}$ is 2linear. Furthermore, the same exact sequence provides for the Hilbert series

$$
P\left(R / I_{\lambda}, t\right)=P\left(R / I_{\lambda^{\prime}}, t\right)-\frac{t^{2}}{(1-t)^{m+1}} .
$$

Using the induction hypothesis, an easy computation provides the claim for the Hilbert series of $R / I_{\lambda}$.

Suppose now $\lambda_{n} \geq 2$ and consider the partition $\lambda^{\prime}=\left(\lambda_{1}, \lambda_{2}, \ldots, \lambda_{n-1}, \lambda_{n}-1\right)$. If in addition we assume that $\lambda_{n} \geq 3$, then the index $s$ of the partition $\lambda^{\prime}$ also equals $n$. The inductive hypothesis provides that a primary decomposition of $I_{\lambda^{\prime}}$ is

$$
\begin{aligned}
& \left(y_{1}, \ldots, y_{\lambda_{1}}\right) \cap\left(x_{1}, y_{1}, \ldots, y_{\lambda_{2}}\right) \cap\left(x_{1}, x_{2}, y_{1}, \ldots, y_{\lambda_{3}}\right) \cap \ldots \\
& \quad \cap\left(x_{1}, \ldots, x_{n-1}, y_{1}, \ldots, y_{\lambda_{n}-1}\right) \cap\left(x_{1}, \ldots, x_{n}, y_{1}\right) \cap\left(x_{1}, \ldots, x_{n}\right) .
\end{aligned}
$$

Let $J$ denote the intersection of all the components in the above primary decomposition that do contain $x_{n} y_{\lambda_{n}}$. Thus, $I_{\lambda^{\prime}}=J \cap\left(x_{1}, \ldots, x_{n-1}, y_{1}, \ldots, y_{\lambda_{n}-1}\right)$. Now observe that $I_{\lambda}=I_{\lambda^{\prime}}+\left(x_{n} y_{\lambda_{n}}\right)$; thus using the above primary decomposition for $I_{\lambda^{\prime}}$, we get

$$
\begin{aligned}
I_{\lambda} & =J \cap\left(x_{1}, \ldots, x_{n-1}, y_{1}, \ldots, y_{\lambda_{n}-1}, x_{n} y_{\lambda_{n}}\right) \\
& =J \cap\left(x_{1}, \ldots, x_{n-1}, y_{1}, \ldots, y_{\lambda_{n}-1}, y_{\lambda_{n}}\right) \cap\left(x_{1}, \ldots, x_{n-1}, y_{1}, \ldots, y_{\lambda_{n}-1}, x_{n}\right) \\
& =J \cap\left(x_{1}, \ldots, x_{n-1}, y_{1}, \ldots, y_{\lambda_{n}}\right),
\end{aligned}
$$

which is, after some inspection, exactly the asserted primary decomposition of $I_{\lambda}$. Turning to the Betti numbers of $I_{\lambda}$, the given primary decomposition implies that $I_{\lambda^{\prime}}: x_{n} y_{\lambda_{n}}=\left(x_{1}, \ldots, x_{n-1}, y_{1}, \ldots, y_{\lambda_{n}-1}\right)$. Hence, by a similar mapping cone argument as above, we obtain that

$$
\begin{aligned}
\beta_{i}\left(R / I_{\lambda}\right) & =\beta_{i}\left(R / I_{\lambda^{\prime}}\right)+\beta_{i-1}\left(R /\left(x_{1}, \ldots, x_{n-1}, y_{1}, \ldots, y_{\lambda_{n}-1}\right)\right) \\
& =\beta_{i}\left(R / I_{\lambda^{\prime}}\right)+\left(\begin{array}{c}
\lambda_{n}-1+n-1 \\
i-1
\end{array}\right)
\end{aligned}
$$

and that the resolution of $R / I_{\lambda}$ is 2-linear. Therefore, using our inductive assumption we get that $\beta_{i}\left(R / I_{\lambda}\right)$ is given by

$\left(\begin{array}{c}\lambda_{1} \\ i\end{array}\right)+\ldots+\left(\begin{array}{c}\lambda_{n-1}+n-2 \\ i\end{array}\right)+\left(\begin{array}{c}\lambda_{n}-1+n-1 \\ i\end{array}\right)-\left(\begin{array}{c}n \\ i+1\end{array}\right)+\left(\begin{array}{c}\lambda_{n}-1+n-1 \\ i-1\end{array}\right)$, 
which can be rewritten in the form

$$
\left(\begin{array}{c}
\lambda_{1} \\
i
\end{array}\right)+\ldots+\left(\begin{array}{c}
\lambda_{n-1}+n-2 \\
i
\end{array}\right)+\left(\begin{array}{c}
\lambda_{n}+n-1 \\
i
\end{array}\right)-\left(\begin{array}{c}
n \\
i+1
\end{array}\right)
$$

as claimed.

To finish our proof, let us assume that $\lambda_{n}=2$ and consider the partition $\lambda^{\prime}=$ $\left(\lambda_{1}, \ldots, \lambda_{n-1}, 1\right)$, whose index $s$ is $n-1$. Moreover, by the inductive assumption we have that a primary decomposition of $I_{\lambda^{\prime}}$ is

$$
\begin{aligned}
& \left(y_{1}, \ldots, y_{\lambda_{1}}\right) \cap\left(x_{1}, y_{1}, \ldots, y_{\lambda_{2}}\right) \cap\left(x_{1}, x_{2}, y_{1}, \ldots, y_{\lambda_{3}}\right) \cap \ldots \\
& \quad \cap\left(x_{1}, \ldots, x_{n-2}, y_{1}, \ldots, y_{\lambda_{n-1}}\right) \cap\left(x_{1}, \ldots, x_{n-1}, y_{1}\right) \cap\left(x_{1}, \ldots, x_{n}\right) .
\end{aligned}
$$

Let $J$ denote the intersection of all the components in the above primary decomposition that contain $x_{n} y_{2}$. Thus, $I_{\lambda^{\prime}}=J \cap\left(x_{1}, \ldots, x_{n-1}, y_{1}\right)$. Now observe that $I_{\lambda}=I_{\lambda^{\prime}}+\left(x_{n} y_{2}\right)$; thus using the above primary decomposition for $I_{\lambda^{\prime}}$, we get

$$
\begin{aligned}
I_{\lambda} & =J \cap\left(x_{1}, \ldots, x_{n-1}, y_{1}, x_{n} y_{2}\right) \\
& =J \cap\left(x_{1}, \ldots, x_{n-1}, y_{1}, y_{2}\right) \cap\left(x_{1}, \ldots, x_{n-1}, y_{1}, x_{n}\right) \\
& =J \cap\left(x_{1}, \ldots, x_{n-1}, y_{1}, y_{2}\right),
\end{aligned}
$$

which is, after some inspection, exactly the asserted primary decomposition of $I_{\lambda}$. The given primary decomposition of $I_{\lambda^{\prime}}$ provides that $I_{\lambda^{\prime}}: x_{n} y_{2}=\left(x_{1}, \ldots, x_{n-1}, y_{1}\right)$. Hence, a mapping cone argument implies that

$$
\beta_{i}\left(R / I_{\lambda}\right)=\beta_{i}\left(R / I_{\lambda^{\prime}}\right)+\beta_{i-1}\left(R /\left(x_{1}, \ldots, x_{n-1}, y_{1}\right)\right)=\beta_{i}\left(R / I_{\lambda^{\prime}}\right)+\left(\begin{array}{c}
n \\
i-1
\end{array}\right) .
$$

Therefore, using our inductive assumption, we see that $\beta_{i}\left(I_{\lambda}\right)$ is given by

$$
\left(\begin{array}{c}
\lambda_{1} \\
i
\end{array}\right)+\ldots+\left(\begin{array}{c}
\lambda_{n-1}+n-2 \\
i
\end{array}\right)+\left(\begin{array}{c}
n \\
i
\end{array}\right)-\left(\begin{array}{c}
n \\
i+1
\end{array}\right)+\left(\begin{array}{c}
n \\
i-1
\end{array}\right)
$$

which can be rewritten in the form

$$
\left(\begin{array}{c}
\lambda_{1} \\
i
\end{array}\right)+\ldots+\left(\begin{array}{c}
\lambda_{n-1}+n-2 \\
i
\end{array}\right)+\left(\begin{array}{c}
n+1 \\
i
\end{array}\right)-\left(\begin{array}{c}
n \\
i+1
\end{array}\right)
$$

which gives the asserted formula as $\left(\begin{array}{c}n+1 \\ i\end{array}\right)=\left(\begin{array}{c}2+\left(\begin{array}{c}n-1) \\ i\end{array}\right) \\ \text {. }\end{array}\right)$.

The claims about the 2-linearity of the resolution and about the Hilbert series follow similarly as in the case $\lambda_{n}=1$. We omit the details.

Theorem 2.1 allows us to compute further invariants of Ferrers ideals:

Corollary 2.2. Adopt the notation of Theorem 2.1. Then the height of the edge ideal $I_{\lambda}$ of a Ferrers graph $G$ is $\min \left\{n, \min _{1 \leq j \leq n}\left\{\lambda_{j}+j-1\right\}\right\}$, the projective dimension of the factor ring $R / I_{\lambda}$ is $\max _{1 \leq j \leq n}\left\{\lambda_{j}+j-1\right\}$ and the Castelnuovo-Mumford regularity $\operatorname{reg}\left(I_{\lambda}\right)$ is equal to 2 .

Proof. It suffices to discuss the claim concerning the projective dimension. Set $i=\max _{1 \leq j \leq n}\left\{\lambda_{j}+j-1\right\}$ and observe that $i \geq n$. Hence the formula in Theorem 2.1 implies $\beta_{i}\left(R / I_{\lambda}\right) \geq 1$. 
Example 2.3. Consider the Ferrers ideal $I_{\lambda}$ with $\lambda=(6,4,4,2,1)$ described in Figure 1. Using our result we get that the projective dimension of $R / I_{\lambda}$ is 6 , the height of $I_{\lambda}$ is 5 , and the minimal free resolution of $R / I_{\lambda}$ is of the form

$$
\begin{aligned}
0 & \rightarrow R^{2}(-7) \longrightarrow R^{15}(-6) \longrightarrow R^{44}(-5) \\
& \longrightarrow R^{65}(-4) \longrightarrow R^{50}(-3) \longrightarrow R^{17}(-2) \longrightarrow R \longrightarrow R / I_{\lambda} \rightarrow 0 .
\end{aligned}
$$

Remark 2.4. By a result of Herzog-Hibi-Zheng [31, Theorem 3.2], all the powers $I_{\lambda}^{k}$ have a linear resolution so that the Castelnuovo-Mumford regularity of $I_{\lambda}^{k}$, for any integer $k \geq 1$, is $\operatorname{reg}\left(I_{\lambda}^{k}\right)=2 k$.

Example 2.5. The edge ideal $I_{\lambda}$ of the complete bipartite graph on $n$ and $m$ vertices, respectively, is $I_{\lambda}=\left(x_{1}, \ldots, x_{n}\right)\left(y_{1}, \ldots, y_{m}\right)=\left(x_{1}, \ldots, x_{n}\right) \cap\left(y_{1}, \ldots, y_{m}\right)$ and has $i$-th Betti number:

$$
\beta_{i}\left(R / I_{\lambda}\right)=\left(\begin{array}{c}
m+n \\
i+1
\end{array}\right)-\left(\begin{array}{c}
m \\
i+1
\end{array}\right)-\left(\begin{array}{c}
n \\
i+1
\end{array}\right)
$$

for $1 \leq i \leq n+m-1$. In fact, the formula for the Betti numbers follows from Theorem 2.1] and [25. Formula (1.48)], or simply by using the Mayer-Vietoris sequence.

The primary decomposition of the Ferrers ideal $I_{\lambda}$ described in Theorem $2.1 \mathrm{can}$ be refined into an irredundant one by using the shape of the Ferrers tableau $\mathbf{T}_{\lambda}$. More precisely, define recursively indices $j_{0}, \ldots, j_{t}$ by setting $j_{0}=0$ and, for $i \geq 0$,

$$
j_{i+1}=\max \left\{k \mid \lambda_{k}=\lambda_{j_{i}+1}\right\} .
$$

Note that $\lambda_{j_{1}}=m, j_{t}=n$, and that the pairs $\left(j_{i}, \lambda_{j_{i}}\right), i=1, \ldots, t$, are the coordinates of the outer corners of the Ferrers tableau $\mathbf{T}_{\lambda}$. In addition, set $\lambda_{j_{t+1}}=0$ and, accordingly, $\left(x_{1}, \ldots, x_{j_{0}}\right)=(0)=\left(y_{1}, \ldots, y_{\lambda_{j_{t+1}}}\right)$. With this notation, we state next our refinement of the primary decomposition described in Theorem 2.1.

Corollary 2.6. The irredundant primary decomposition of the Ferrers ideal $I_{\lambda}$ is

$$
I_{\lambda}=\bigcap_{i=1}^{t+1}\left(x_{1}, \ldots, x_{j_{i-1}}, y_{1}, \ldots, y_{\lambda_{j_{i}}}\right),
$$

where the pairs $\left(j_{i}, \lambda_{j_{i}}\right), i=1, \ldots, t$, correspond to the $t$ outer corners of the Ferrers tableau of $I_{\lambda}$. In particular, $I_{\lambda}$ is the intersection of $t+1$ prime ideals.

Proof. This follows by inspecting the decomposition given in Theorem 2.1

Corollary 2.7. Adopt the notation of Theorem 2.1 as well as the one established above. Then the following conditions are equivalent:

(a) $I_{\lambda}$ is unmixed;

(b) $n=m$ and the inside corners $\left(j_{i-1}, \lambda_{j_{i}}\right)$, for $i=2, \ldots$, $t$, of the Ferrers tableau $\mathbf{T}_{\lambda}$ of $I_{\lambda}$ lie on the main anti-diagonal of $\mathbf{T}_{\lambda}$, i.e. on $\{(p, q) \mid p+q=$ $m\}$.

Proof. The equivalence of the conditions follows immediately from Corollary 2.6.

Ferrers ideals are rarely Cohen-Macaulay. In fact, we get:

Corollary 2.8. The following conditions are equivalent:

(a) $I_{\lambda}$ is unmixed and connected in codimension one; 
(b) $n=m$ and $\lambda=(n, n-1, n-2, \ldots, 3,2,1)$;

(c) $I_{\lambda}$ is a Cohen-Macaulay ideal.

In particular, in this case the $i$-th Betti number is:

$$
\beta_{i}\left(R / I_{\lambda}\right)=i\left(\begin{array}{c}
n+1 \\
i+1
\end{array}\right)
$$

for $1 \leq i \leq n(=m)$. Moreover, the Cohen-Macaulay type is $n$ and the Hilbert series $P\left(R / I_{\lambda}, t\right)$ is given by

$$
P\left(R / I_{\lambda}, t\right)=\frac{1+n t}{(1-t)^{n}} .
$$

Proof. Corollary 2.2 shows that $(b)$ implies $(c)$. Condition $(a)$ is always a consequence of $(c)$. Using Corollary 2.6, we see that $(a)$ implies $(b)$.

Concerning the Betti numbers of $I_{\lambda}$, Theorem 2.1 and the shape of the partition $\lambda$ provide that

$$
\beta_{i}\left(R / I_{\lambda}\right)=n\left(\begin{array}{c}
n \\
i
\end{array}\right)-\left(\begin{array}{c}
n \\
i+1
\end{array}\right)
$$

for $1 \leq i \leq n$. On the other hand, an easy calculation shows that the latter expression equals $i\left(\begin{array}{c}n+1 \\ i+1\end{array}\right)$, as claimed. The statement concerning the Hilbert series follows immediately from Theorem 2.1.

We note that the equivalence of conditions $(b)$ and $(c)$ above can also be deduced from a recent result of Herzog and Hibi [30, Theorem 3.4]. In general, the condition that a projective subscheme $Z \subset \mathbb{P}^{n}$ is equidimensional and connected in codimension one is only a necessary condition for $Z$ being arithmetically Cohen-Macaulay. However, if $Z$ is defined by a monomial ideal that has a combinatorial interpretation, then it seems often the case that this condition is also sufficient. Note however that it is not always true. For example, if $Z \subset \mathbb{P}^{4}$ is defined by $(x y, x z w, y w t, z w t)$, then $Z$ is connected in codimension 1 , but $Z$ is not arithmetically Cohen-Macaulay.

\section{Minimal free Resolutions of Ferrers ideals}

In this section we explicitly describe the minimal free resolution of every Ferrers ideal. For our construction we use cellular resolutions and polyhedral cell complexes as introduced by Bayer and Sturmfels in 3 . First, we briefly recall some basic notions, but we refer to [3] (or [41]) for a more detailed introduction to the topic. A polyhedral cell complex $X$ is a finite collection of convex polytopes (in some $\mathbb{R}^{N}$ ) called faces (or cells) of $X$ such that:

(1) if $P \in X$ and $F$ is a face of $P$, then $F \in X$;

(2) if $P, Q \in X$, then $P \cap Q$ is a face of both $P$ and $Q$.

Let $F_{k}(X)$ be the set of $k$-dimensional faces. Each cell complex admits an incidence function $\varepsilon$ on $X$ where $\varepsilon(Q, P) \in\{1,-1\}$ if $Q$ is a facet of $P \in X . X$ is called a labeled cell complex if each vertex $i$ has a vector $\mathbf{a}_{i} \in \mathbb{N}^{N}$ (or the monomial $\mathbf{z}^{\mathbf{a}_{i}}$, where $\mathbf{z}^{\mathbf{a}_{i}}$ denotes a monomial in the variables $\left.z_{1}, \ldots, z_{N}\right)$ as label. The label of an arbitrary face $Q$ of $X$ is the exponent $\mathbf{a}_{Q}$, where $\mathbf{z}^{\mathbf{a}_{Q}}:=\operatorname{lcm}\left(\mathbf{z}^{\mathbf{a}_{i}} \mid i \in Q\right)$. Each labeled cell complex determines a complex of free $R$-modules, where $R=$ $K\left[z_{1}, \ldots, z_{N}\right]$. The cellular complex $\mathcal{F}_{X}$ supported on $X$ is the complex of free $\mathbb{Z}^{N}$-graded $R$-modules

$$
\mathcal{F}_{X}: \quad 0 \rightarrow S^{F_{d}(X)} \stackrel{\partial_{d}}{\longrightarrow} S^{F_{d-1}(X)} \stackrel{\partial_{d-1}}{\longrightarrow} \cdots \stackrel{\partial_{2}}{\longrightarrow} S^{F_{1}(X)} \stackrel{\partial_{1}}{\longrightarrow} S^{F_{0}(X)} \stackrel{\partial_{0}}{\longrightarrow} S \rightarrow 0,
$$


where $d=\operatorname{dim} X$ and $S^{F_{k}(X)}:=\bigoplus_{P \in F_{k}(X)} R\left[-\mathbf{a}_{P}\right]$. The map $\partial_{k}$ is defined by

$$
\partial_{k}\left(e_{P}\right):=\sum_{Q \text { facet of } P} \varepsilon(P, Q) \cdot \mathbf{z}^{\mathbf{a}_{P}-\mathbf{a}_{Q}} \cdot e_{Q},
$$

where $\left\{e_{P} \mid P \in F_{k}(X)\right\}$ is a basis of $S^{F_{k}(X)}$ and $e_{\emptyset}:=1$. If $\mathcal{F}_{X}$ is acyclic, then it provides a free $\mathbb{Z}^{N}$-graded resolution of the image $I$ of $\partial_{0}$, that is, the ideal generated by the labels of the vertices of $X$. In this case, $\mathcal{F}_{X}$ is called a cellular resolution of $I$.

We are ready to describe a cellular minimal free resolution for each Ferrers ideal. First, let us consider the complete bipartite graph $\mathcal{K}_{n, m}$ that corresponds to the edge ideal $\left(x_{1}, \ldots, x_{n}\right)\left(y_{1}, \ldots, y_{m}\right)$ (or the partition $\lambda$, where $\lambda_{i}=m$ for $i=1, \ldots, n$ ). To this graph, we associate the polyhedral cell complex $X_{n, m}$ given by the face complex of the polytope $\Delta_{n-1} \times \Delta_{m-1}$ obtained by taking the cartesian product of the $(n-1)$-simplex $\Delta_{n-1}$ and the $(m-1)$-simplex $\Delta_{m-1}$. Labeling the vertices of $\Delta_{n-1}$ by $x_{1}, \ldots, x_{n}$ and the ones of $\Delta_{m-1}$ by $y_{1}, \ldots, y_{m}$, the vertices of the cell complex $X_{n, m}$ are naturally labeled by the monomials $x_{i} y_{j}$ with $1 \leq i \leq n$ and $1 \leq j \leq m$. An easy example is illustrated in Figure 3.

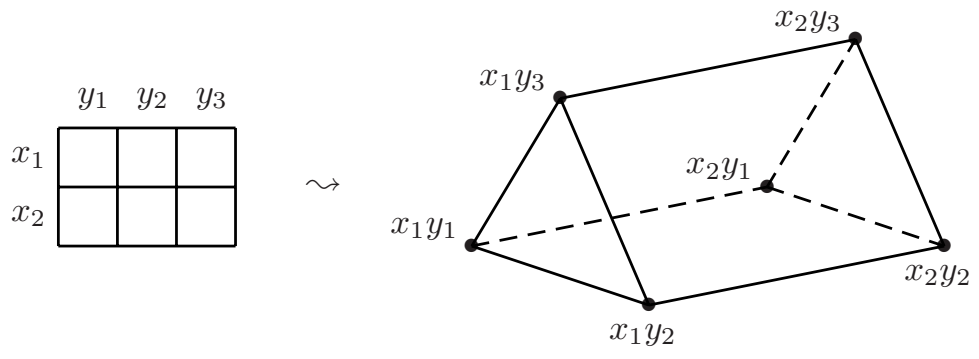

Figure 3. Topological viewpoint, $\Delta_{1} \times \Delta_{2}$

To simplify notation, we denote the monomial that labels the face $P \in X_{n, m}$ by $m_{P}$. Recall that $m_{p}$ is the least common multiple of the monomial labels of the vertices of $P$. In general, we observe that $\operatorname{deg} m_{P}=\operatorname{dim} P+2$ for each face $P \in X_{n, m}$.

We are now in a position to define the cell complex that will support the cellular resolution of a given Ferrers ideal. As above, we fix a partition $\lambda=\left(\lambda_{1}, \lambda_{2}, \ldots, \lambda_{n}\right)$ with $\lambda_{1}=m$, corresponding to a Ferrers graph $G_{\lambda}$ and a Ferrers ideal $I_{\lambda} \subset R=$ $K\left[x_{1}, \ldots, x_{n}, y_{1}, \ldots, y_{m}\right]$.

Definition 3.1. The polyhedral cell complex $X_{\lambda}$ associated to the partition $\lambda$ is the labeled subcomplex of $X_{n, m}$ consisting of the faces of $X_{n, m}$ whose vertices are labeled by all the monomials generating the Ferrers ideal $I_{\lambda}$.

Using the Ferrers tableau $\mathbf{T}_{\lambda}$ we get a more explicit, yet simple, description of the cell complex $X_{\lambda}$. In fact, it is easy to see that the facets of $X_{\lambda}$ are in oneto-one correspondence with the outer corners of $\mathbf{T}_{\lambda}$. More precisely, if $\left(i, \lambda_{i}\right)$ is an outer corner of $\mathbf{T}_{\lambda}$, then the product of the polytopes (simplices) with vertices $\left\{x_{1}, \ldots, x_{i}\right\}$ and $\left\{y_{1}, \ldots, y_{\lambda_{i}}\right\}$ is a facet of $X_{\lambda}$. Each facet of $X_{\lambda}$ determines a 
rectangular region in the Ferrers tableau $\mathbf{T}_{\lambda}$. The intersection of the regions corresponding to two facets is again a rectangle that corresponds to a product of smaller simplices. This product polytope is the intersection of the two facets of $X_{\lambda}$. An example is illustrated in Figure 4.
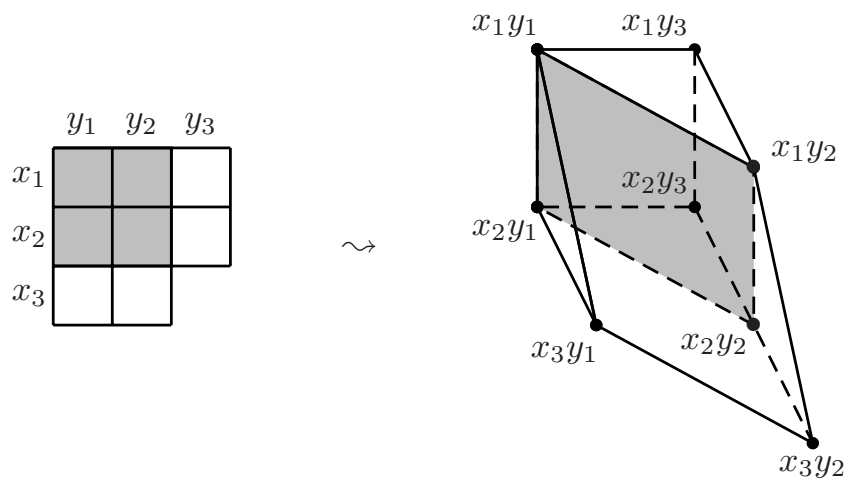

Figure 4 . Faces of the polyhedral cell complex $X_{\lambda}$

The main result of this section is:

Theorem 3.2. The complex $\mathcal{F}_{X_{\lambda}}$ provides the minimal free $\mathbb{Z}^{m+n}$-graded resolution of $I_{\lambda}$.

It is clear that $\mathcal{F}_{X_{\lambda}}$ also gives a $\mathbb{Z}$-graded minimal free resolution of $I_{\lambda}$. In fact, since the label of each $k$-dimensional face of $X_{\lambda}$ has degree $k+2$ as noted above,

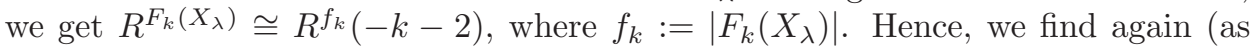
seen in Theorem 2.1) that the $\mathbb{Z}$-graded minimal free resolution of $I_{\lambda}$ is 2-linear.

Proof. For fixed $m=\lambda_{1}$, we will induct on $|\lambda|=\lambda_{1}+\ldots+\lambda_{n} \geq m$. If $|\lambda|=m$, then $X_{\lambda}=X_{1, m}$ and the claim follows from the discussion of complete bipartite graphs above Definition 3.1. Let $|\lambda|>m$. We divide the argument into five steps:

(I) For each $k \leq n+\lambda_{n}-2$, let $G_{k-1} \subset R^{F_{k}\left(X_{\lambda}\right)}$ denote the free $R$-module generated by the $k$-dimensional faces of $X_{\lambda}$ involving the vertex $x_{n} y_{\lambda_{n}}$. Its rank is

$$
\operatorname{rank} G_{k-1}=\left(\begin{array}{c}
n+\lambda_{n}-2 \\
k
\end{array}\right) \text {. }
$$

Indeed, each such face corresponds to the boxes lying on a suitable grid of $a$ rows and $b$ columns indexed by $i_{1}<i_{2}<\cdots<i_{a}=n$ and $j_{1}<j_{2}<\cdots<j_{b}=\lambda_{n}$, where $a+b-2=k$. In this way, we see that the number of such $k$-dimensional faces is

$$
\begin{aligned}
\operatorname{rank} G_{k-1} & =\sum_{a=1}^{k+1}\left(\begin{array}{c}
n-1 \\
a-1
\end{array}\right)\left(\begin{array}{c}
\lambda_{n}-1 \\
b-1
\end{array}\right)=\sum_{a=1}^{k+1}\left(\begin{array}{c}
n-1 \\
a-1
\end{array}\right)\left(\begin{array}{c}
\lambda_{n}-1 \\
k-a+1
\end{array}\right) \\
& =\sum_{j=0}^{k}\left(\begin{array}{c}
n-1 \\
j
\end{array}\right)\left(\begin{array}{c}
\lambda_{n}-1 \\
k-j
\end{array}\right)=\left(\begin{array}{c}
n+\lambda_{n}-2 \\
k
\end{array}\right) .
\end{aligned}
$$

The latter equality is nothing but the Vandermonde convolution [25, Formula 3.1]. 
(II) The proof of Theorem 2.1 shows that there is a partition $\lambda^{\prime}$ such that $\left|\lambda^{\prime}\right|=|\lambda|-1$

$$
I_{\lambda}=I_{\lambda^{\prime}}+\left(x_{n} y_{\lambda_{n}}\right) \quad \text { and } \quad I_{\lambda^{\prime}}: x_{n} y_{\lambda_{n}}=\left(x_{1}, \ldots, x_{n-1}, y_{1}, \ldots, y_{\lambda_{n}-1}\right) \text {. }
$$

This provides the exact sequence

$$
0 \rightarrow R /\left(x_{1}, \ldots, x_{n-1}, y_{1}, \ldots, y_{\lambda_{n}-1}\right)[-2] \stackrel{x_{n} y_{\lambda_{n}}}{\longrightarrow} R / I_{\lambda^{\prime}} \longrightarrow R / I_{\lambda} \rightarrow 0 .
$$

(III) Let $\varepsilon$ be the incidence function of $X_{\lambda}$ that gives the signs in $\mathcal{F}_{X_{\lambda}}$. Then its restriction to $X_{\lambda^{\prime}}$ is an incidence function too, which we use to define the cell complex $\mathcal{F}_{X_{\lambda^{\prime}}}$.

Observe that, for each variable $l \in R$ and each non-empty face $P \in X_{\lambda}$, there is a unique facet $Q$ of $P$ such that $m_{P}=l \cdot m_{Q}$. We denote this facet $Q$ by $P / l$. Let $P$ denote a $k$-dimensional face of $X_{\lambda}$ involving the monomial $x_{n} y_{\lambda_{n}}$ and observe that $\partial_{k}\left(e_{P}\right)$ can be written as

$$
\begin{aligned}
\partial_{k}\left(e_{P}\right) & =\sum_{l \mid m_{P}} l \cdot \varepsilon(P, P / l) e_{P / l} \\
& =\sum_{\substack{l \mid m_{P} \\
l \nmid x_{n} y_{\lambda_{n}}}} l \cdot \varepsilon(P, P / l) e_{P / l}+x_{n} \varepsilon\left(P, P / x_{n}\right) e_{P / x_{n}}+y_{\lambda_{n}} \varepsilon\left(P, P / y_{\lambda_{n}}\right) e_{P / y_{\lambda_{n}}} \\
& =\varphi_{k-1}\left(e_{P}\right)+(-1)^{k} \delta_{k-1}\left(e_{P}\right),
\end{aligned}
$$

where

$$
\begin{aligned}
\varphi_{k-1}\left(e_{P}\right) & =\sum_{\substack{l \mid m_{P} \\
l \nmid x_{n} y_{\lambda_{n}}}} l \cdot \varepsilon(P, P / l) e_{P / l}, \\
\delta_{k-1}\left(e_{P}\right) & =(-1)^{k} x_{n} \varepsilon\left(P, P / x_{n}\right) e_{P / x_{n}}+(-1)^{k} y_{\lambda_{n}} \varepsilon\left(P, P / y_{\lambda_{n}}\right) e_{P / y_{\lambda_{n}}} .
\end{aligned}
$$

Note that $\varphi_{k-1}\left(e_{P}\right)$ is in $G_{k-2}$. Thus, we get a sequence of graded $R$-modules:

$$
\mathbb{G}_{\bullet}: \quad 0 \longrightarrow G_{n+\lambda_{n}-3} \stackrel{\varphi_{n+\lambda_{n}-3}}{\longrightarrow} \ldots \longrightarrow G_{1} \stackrel{\varphi_{1}}{\longrightarrow} G_{0} \longrightarrow 0
$$

where the image of $\varphi_{1}$ is the ideal $\left(x_{1}, \ldots, x_{n-1}, y_{1}, \ldots, y_{\lambda_{n}-1}\right)$. In fact, it is not too difficult to see that $\mathbb{G}_{\bullet}$ is actually the Koszul complex on $x_{1}, \ldots, x_{n-1}, y_{1}, \ldots, y_{\lambda_{n}-1}$, where the degrees are shifted by -2 .

(IV) Set $H_{k}^{\prime}=R^{F_{k}\left(X_{\lambda^{\prime}}\right)}$. Then $R^{F_{k}\left(X_{\lambda}\right)}=H_{k}^{\prime} \oplus G_{k-1}$. Moreover, for each generator $e_{P} \in G_{k-1}, \delta_{k-1}\left(e_{P}\right)$ is in $H_{k-1}^{\prime}$. Hence, we get the following square:

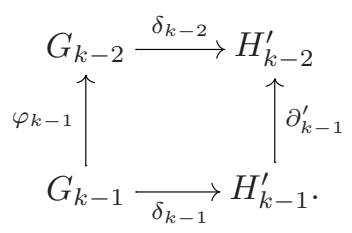


We claim that it is commutative, i.e. $\delta_{k-2} \circ \varphi_{k-1}=\partial_{k-1}^{\prime} \circ \delta_{k-1}$. Indeed, we have that

$$
\begin{aligned}
\delta_{k-2}\left(\varphi_{k-1}\left(e_{P}\right)\right)= & \delta_{k-2}\left(\sum_{l \mid \frac{m_{P}}{x_{n} y_{\lambda_{n}}}} l \varepsilon(P, P / l) e_{P / l}\right) \\
= & (-1)^{k-1}\left(\sum_{l \mid \frac{m_{P}}{x_{n} y_{\lambda_{n}}}} x_{n} l \varepsilon(P, P / l) \varepsilon\left(P / l, P / l x_{n}\right) e_{P / l x_{n}}\right. \\
& \left.+y_{\lambda_{n}} l \varepsilon(P, P / l) \varepsilon\left(P / l, P / l y_{\lambda_{n}}\right) e_{P / l y_{\lambda_{n}}}\right) .
\end{aligned}
$$

On the other hand, we get

$$
\left.\begin{array}{rl}
\partial_{k-1}^{\prime}\left(\delta_{k-1}\left(e_{P}\right)\right) & \partial_{k-1}^{\prime}\left((-1)^{k} x_{n} \varepsilon\left(P, P / x_{n}\right) e_{P / x_{n}}+(-1)^{k} y_{\lambda_{n}} \varepsilon\left(P, P / y_{\lambda_{n}}\right) e_{P / y_{\lambda_{n}}}\right) \\
= & (-1)^{k} x_{n} \varepsilon\left(P, P / x_{n}\right) \sum_{l \mid \frac{m_{P}}{x_{n}}} l \varepsilon\left(P / x_{n}, P / l x_{n}\right) e_{P / l x_{n}} \\
+(-1)^{k} y_{\lambda_{n}} \varepsilon\left(P, P / y_{\lambda_{n}}\right) \sum_{l \mid \frac{m_{P}}{y_{\lambda_{n}}}} l \varepsilon\left(P / y_{\lambda_{n}}, P / l y_{\lambda_{n}}\right) e_{P / l y_{\lambda_{n}}} & \left.+y_{\lambda_{n}} \varepsilon\left(P / x_{n}, P / x_{n} y_{\lambda_{n}}\right) e_{P / x_{n} y_{\lambda_{n}}}\right) \\
= & (-1)^{k} x_{n} \varepsilon\left(P, P / x_{n}\right)\left(\sum_{l \mid \frac{m_{P}}{x_{n} y_{\lambda_{n}}}} l \varepsilon\left(P / x_{n}, P / l x_{n}\right) e_{P / l x_{n}}\right. \\
+(-1)^{k} y_{\lambda_{n}} \varepsilon\left(P, P / y_{\lambda_{n}}\right)\left(\sum_{l \mid \frac{m_{P}}{x_{n} y_{\lambda_{n}}}} l \varepsilon\left(P / y_{\lambda_{n}}, P / l y_{\lambda_{n}}\right) e_{P / l y_{\lambda_{n}}}\right. \\
=(-1)^{k} x_{n} \varepsilon\left(P, P / x_{n}\right)\left(\sum_{l \mid \frac{m_{P}}{x_{n} y_{\lambda_{n}}}} l \varepsilon\left(P / x_{n}, P / l x_{n}\right) e_{P / l x_{n}}\right) \\
+(-1)^{k} y_{\lambda_{n}} \varepsilon\left(P, P / y_{\lambda_{n}}\right)\left(\sum_{l \mid \frac{m_{P}}{x_{n} y_{\lambda_{n}}}} l \varepsilon\left(P / y_{\lambda_{n}}, P / l y_{\lambda_{n}}\right) e_{P / l y_{\lambda_{n}}}\right.
\end{array}\right) .
$$

Observe that the last two equalities follow from one of the properties of incidence functions:

$$
\varepsilon(F, F / l) \cdot \varepsilon(F / l, F / l h)+\varepsilon(F, F / h) \cdot \varepsilon(F / h, F / l h)=0 .
$$


(V) By Step (IV), $\delta_{\bullet}: \mathbb{G}_{\bullet} \rightarrow \mathcal{F}_{X_{\lambda^{\prime}}}$ is a morphism of chain complexes. It allows us to apply the mapping cone procedure to the exact sequence in Step (II), which provides the desired free resolution of $R / I_{\lambda}$.

Remark 3.3. In [20, Eisenbud, Green, Hulek, and Popescu consider more generally a projective subscheme that is the union of linear subspaces and that has a 2-linear free resolution. They construct a free resolution of such a scheme $X$. However, it is not in general minimal though it gives the exact number of minimal generators of the homogeneous ideal $I_{X}$. Our Theorem 3.2 treats the special case where $I_{X}$ is a monomial ideal, but our conclusion is stronger.

\section{Characterization of Ferrers graphs}

In this brief section we establish an intrinsic characterization of Ferrers graphs (not referring to a suitable labeling) by proving the converse of Theorem 2.1. In other words, we characterize Ferrers ideals as essentially the only edge ideals with a 2-linear free resolution among the ones arising from bipartite graphs. To this end we will use a result of Fröberg, which has been recently refined in 19.

Let $G$ be a finite graph on the vertex set $V=\left\{v_{1}, \ldots, v_{n}\right\}$. We recall that the complementary graph $\bar{G}$ of $G$ is the graph (on the same vertex set $V$ as $G$ ) such that, for vertices $v_{i}, v_{j} \in V$, the pair $\left(v_{i}, v_{j}\right)$ is an edge of $\bar{G}$ if and only if $\left(v_{i}, v_{j}\right)$ is not an edge of $G$. Furthermore, the graph $G$ is called chordal if every cycle of $\bar{G}$ of length at least 4 has a chord. With this notation, a result of Fröberg says:

Theorem 4.1 (Fröberg [21]). The edge ideal of a graph $G$ has a 2-linear free resolution if and only if the complementary graph $\bar{G}$ is chordal.

Note that adding an isolated vertex to a given graph $G$ does not change the generating set nor the graded Betti numbers of the edge ideal of $G$. Thus, it is harmless to assume that the graph does not have isolated vertices. We are now ready to show:

Theorem 4.2. Let $G$ be a bipartite graph without isolated vertices. Then its edge ideal has a 2-linear free resolution if and only if $G$ is (up to a relabeling of the vertices) a Ferrers graph.

Proof. We have shown in Theorem 2.1 that the condition is sufficient. We now establish its necessity. Thus, let $G$ be a bipartite graph on two distinct sets of vertices, say $\left\{x_{1}, \ldots, x_{n}\right\}$ and $\left\{y_{1}, \ldots, y_{m}\right\}$, and assume that its edge ideal has a 2-linear resolution. Let $\lambda_{i}$ be the degree of $x_{i}$. By relabeling the vertices, we may also assume that $m \geq \lambda_{1} \geq \lambda_{2} \geq \ldots \geq \lambda_{n} \geq 1$ and that the edges connected to $x_{1}$ are labeled $y_{1}, \ldots, y_{\lambda_{1}}$.

For $1 \leq i \leq n$, we now claim first that the $\lambda_{i}$ vertices connected to $x_{i}$ are among the vertices in $\left\{y_{1}, \ldots, y_{\lambda_{i-1}}\right\}$ and second that in fact they are exactly the first consecutive $\lambda_{i}$ vertices contained in $\left\{y_{1}, \ldots, y_{\lambda_{i-1}}\right\}$. Indeed, there is nothing to prove if $i=1$. Let $i>1$ and first assume that $x_{i}$ is connected to some $y_{k}$ with $k>\lambda_{i-1}$. Thus there exists some $j$, with $1 \leq j \leq \lambda_{i-1}$, such that $x_{i} y_{j}$ is not an edge in $G$. Moreover, the induction hypothesis provides that $x_{i-1} y_{k}$ is not an edge of $G$ either. It follows that the complementary graph $\bar{G}$ contains the cycle $\Gamma=\left\{x_{i} y_{j}, x_{i-1} y_{k}, x_{i-1} x_{i}, y_{j} y_{k}\right\}$ of length 4 . However, none of the chords of $\Gamma$, namely $x_{i} y_{k}$ and $x_{i-1} y_{j}$, belongs to $\bar{G}$. This contradicts Fröberg's theorem. Now, 
by relabeling the vertices of $G$, we may assume that the vertices connected to $x_{i}$ are exactly the first consecutive $\lambda_{i}$ vertices contained in $\left\{y_{1}, \ldots, y_{\lambda_{i-1}}\right\}$.

As a by-product of our argument, we observe that $\lambda_{1}$ is exactly $m$. It also shows that if $\left(x_{p}, y_{q}\right)$ is an edge of $G$, then so is $\left(x_{h}, y_{k}\right)$, provided $1 \leq h \leq p$ and $1 \leq k \leq q$. In addition, $\left(x_{1}, y_{m}\right)$ and $\left(x_{n}, y_{1}\right)$ are edges of $G$. Hence $G$ is a Ferrers graph as claimed.

The following example shows that there are edge ideals with a 2-linear resolution which do not arise from a bipartite graph. However, this ideal can be obtained as a specialization of a suitable Ferrers ideal.

Example 4.3. Let $R=K\left[x_{1}, x_{2}, x_{3}\right]$ be a polynomial ring over a field $K$ and let $I$ be the edge ideal corresponding to a cycle of length three, that is, $I=$ $\left(x_{1} x_{2}, x_{1} x_{3}, x_{2} x_{3}\right)$. Clearly, the ideal $I$ does not arise from a bipartite graph. On the other hand, it is a height two Cohen-Macaulay ideal with the following 2-linear resolution:

$$
0 \rightarrow R^{2}[-3] \stackrel{\varphi}{\longrightarrow} R^{3}[-2] \longrightarrow R \longrightarrow R / I \rightarrow 0 .
$$

We notice though that it can be obtained as a specialization of the Ferrers ideal $I_{\lambda}=\left(x_{1} y_{1}, x_{1} y_{2}, x_{2} y_{1}\right)$, by setting $y_{1}:=x_{3}$ and $y_{2}:=x_{2}$ (see [13]).

Notice that the combination of Theorems 4.2 and 2.1 provides a complete description of the possible Betti numbers of edge ideals of bipartite graphs with a 2-linear free resolution.

\section{Toric Rings associated to Ferrers ideals}

Let $\mathbf{T}:=\mathbf{T}_{\lambda}$ denote the Ferrers tableau associated to a Ferrers graph $G$ with partition $\lambda=\left(\lambda_{1}, \ldots, \lambda_{s}, 1, \ldots, 1\right)$. We now define an associated tableau $\mathbf{T}^{\prime}$ obtained from $\mathbf{T}$ by deleting all boxes in the first row beyond the $\lambda_{2}$ one, and all boxes in the first column beyond the $s$ one. Hence the partition $\lambda^{\prime}$ associated to $\mathbf{T}^{\prime}$ is $\left(\lambda_{2}, \lambda_{2}, \lambda_{3}, \ldots, \lambda_{s}\right)$. Observe that, in this manner, the thickness of the outer border of $\mathbf{T}^{\prime}$ is at least 2. From a combinatorial point of view, we removed from $G$ all the vertices (and, a fortiori, the corresponding edges) having degree 1. An example is illustrated in Figure 5.

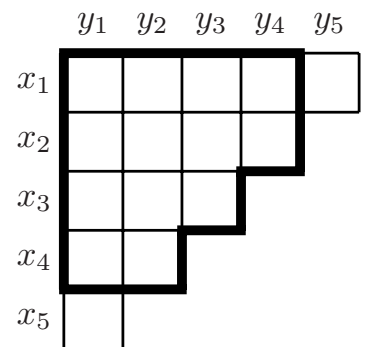

Ferrers tableau $\mathbf{T}$

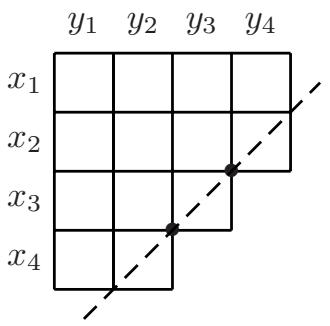

Ferrers tableau $\mathbf{T}^{\prime}$

Figure 5. Ferrers tableaux $\mathbf{T}$ and $\mathbf{T}^{\prime}$ 
According to [46, the Rees algebra $R[I t]$, the associated graded ring $\operatorname{gr}_{I}(R)$ and the special fiber ring $\mathcal{F}(I)$ of the edge ideal $I$ of every bipartite graph are normal Cohen-Macaulay domains. Since edge ideals are generated in one degree, the special fiber ring is also isomorphic to the toric ring of the graph.

Proposition 5.1. Let $\mathbf{X}=\left\{x_{1}, \ldots, x_{n}\right\}$ and $\mathbf{Y}=\left\{y_{1}, \ldots, y_{m}\right\}$ be distinct sets of variables. Set $R=K[\mathbf{X}, \mathbf{Y}]$, where $K$ is a field, and let $I_{\lambda}$ be the edge ideal corresponding to a Ferrers graph $G_{\lambda}$ with associated tableaux $\mathbf{T}$ and $\mathbf{T}^{\prime}$, and partition $\lambda=\left(\lambda_{1}, \lambda_{2}, \ldots, \lambda_{s}, 1, \ldots, 1\right)$. Then the special fiber ring $\mathcal{F}\left(I_{\lambda}\right)$ of $I_{\lambda}$ has the following properties:

(a) $\mathcal{F}\left(I_{\lambda}\right)$ is a Cohen-Macaulay normal domain of dimension $n+m-1$;

(b) $\mathcal{F}\left(I_{\lambda}\right)$ is the ladder determinantal ring $k[\mathbf{T}] / I_{2}\left(\mathbf{T}^{\prime}\right)$;

(c) $\mathcal{F}\left(I_{\lambda}\right)$ is Gorenstein if and only if $\lambda_{2}=s$ and all the inside corners (if any) of the Ferrers tableau $\mathbf{T}^{\prime}$ lie on the main anti-diagonal of $\mathbf{T}^{\prime}$, i.e. $\left\{(i, j) \in \mathbf{T}^{\prime} \mid i+j=\lambda_{2}+1\right\}$.

Proof. The result stated in $(a)$ is due to Simis, Vasconcelos and Villarreal and holds for the special fiber ring of the edge ideal of every connected bipartite graph [46]. It is also recovered by part $(b)$, as ladder determinantal rings are known to have such properties (see [43, 32, 10]).

In order to prove $(b)$, observe that $\mathcal{F}\left(I_{\lambda}\right) \cong K\left[x_{i} y_{j}{ }^{\prime} \mathrm{s}\right]=K\left[G_{\lambda}\right]$, as $I_{\lambda}$ is generated by homogeneous polynomials of the same degree. Moreover, since $G_{\lambda}$ is a bipartite graph its dimension is $m+n-1$ (see [46] or [51, 8.2.13]). Let $\mathbf{T}$ and $\mathbf{T}^{\prime}$ denote the Ferrers tableaux associated to $G_{\lambda}$. Let $T_{i j}$, for $(i, j) \in \mathbf{T}$, be distinct variables: each variable is associated to the corresponding box of the tableau $\mathbf{T}$ (and $\mathbf{T}^{\prime}$, respectively). By abuse of notation we also let $\mathbf{T}$ (and $\mathbf{T}^{\prime}$, respectively) denote the collection of these new variables. We now consider the following epimorphism

$$
\pi: K[\mathbf{T}] \rightarrow K\left[G_{\lambda}\right] \cong \mathcal{F}\left(I_{\lambda}\right),
$$

where $\pi\left(T_{i j}\right)=x_{i} y_{j}$. We claim that the kernel of $\pi$ is the determinantal ideal $I_{2}\left(\mathbf{T}^{\prime}\right)=I_{2}\left(\mathbf{T}^{\prime}\right) \cdot k[\mathbf{T}]$ generated by the $2 \times 2$ minors of the one-sided ladder $\mathbf{T}^{\prime}$. It is clear that the ideal $I_{2}\left(\mathbf{T}^{\prime}\right) \cdot k[\mathbf{T}]$ is contained in the ideal $\operatorname{ker}(\pi)$. On the other hand, we now show that these ideals have the same height. Hence they coincide, as they are both prime ideals (see 43 for the primeness of $I_{2}\left(\mathbf{T}^{\prime}\right)$ ). Indeed, we have

$$
\begin{aligned}
\text { ht } \operatorname{ker}(\pi) & =\operatorname{dim} k[\mathbf{T}]-\operatorname{dim} k\left[G_{\lambda}\right] \\
& =\left(\lambda_{1}+\lambda_{2}+\cdots+\lambda_{s}+n-s\right)-(n+m-1) \\
& =\lambda_{2}+\cdots+\lambda_{s}-s+1
\end{aligned}
$$

(as $\lambda_{1}=m$ ), whereas

$$
\text { ht } \begin{aligned}
I_{2}\left(\mathbf{T}^{\prime}\right) \cdot k[\mathbf{T}] & =\text { ht } I_{2}\left(\mathbf{T}^{\prime}\right) \cdot k\left[\mathbf{T}^{\prime}\right] \\
& =\operatorname{dim} k\left[\mathbf{T}^{\prime}\right]-\operatorname{dim} R_{2}\left(\mathbf{T}^{\prime}\right) \\
& =\lambda_{2}+\lambda_{2}+\cdots+\lambda_{s}-\left(s+\lambda_{2}-1\right) \\
& =\lambda_{2}+\cdots+\lambda_{s}-s+1 .
\end{aligned}
$$

As far as $(c)$ is concerned, the Gorensteiness of $\mathcal{F}\left(I_{\lambda}\right)$ now follows from work of Conca [10, 2.5].

Corollary 5.2. The special fiber ring $\mathcal{F}\left(I_{\lambda}\right)$ is Gorenstein if and only if there is a partition $\mu$ such that the Ferrers ideal $I_{\mu}$ is unmixed and there are variables such that the polynomial rings over $\mathcal{F}\left(I_{\lambda}\right)$ and $\mathcal{F}\left(I_{\mu}\right)$, respectively, are isomorphic. 
Proof. Assume that $\mathcal{F}\left(I_{\lambda}\right)$ is Gorenstein. Then define $\mu:=\left(\lambda_{2}+1, \lambda_{2}, \ldots, \lambda_{s}, 1\right) \in$ $\mathbb{Z}^{\lambda_{2}+1}$. It follows that the ladder determinantal ideals determined by $\lambda$ and $\mu$, respectively, have the same generators. Moreover, Proposition 5.1 and Corollary 2.7 provide that $I_{\mu}$ is unmixed.

Conversely, if $I_{\mu}$ is unmixed, then we see that $\mathcal{F}\left(I_{\mu}\right)$ is Gorenstein using first Corollary 2.7 and then Proposition 5.1 .

As announced earlier, we now turn our attention to the computation of the Hilbert series of the toric ring $K\left[G_{\lambda}\right]$. This is a highly investigated area of research; see, for example, 11, 8, 10, 11, 12, 26, 32, 34, 35, 36, 37, 38, 43, 45, 52. While most of these works involve - to a different extent - path counting arguments, we offer here a new and self-contained approach based on Gorenstein liaison theory. Proposition 5.11implies that, for each partition $\lambda \in \mathbb{N}^{n}$, there is a unique polynomial $p_{\lambda} \in \mathbb{Z}[t]$ such that the Hilbert series of $K\left[G_{\lambda}\right] \cong \mathcal{F}\left(I_{\lambda}\right)$ can be written as

$$
P\left(K\left[G_{\lambda}\right], t\right)=\frac{p_{\lambda}(t)}{(1-t)^{n+m-1}} .
$$

Note that the multiplicity of $K\left[G_{\lambda}\right]$ is $e\left(K\left[G_{\lambda}\right]\right)=p_{\lambda}(1)$. With this notation and using Gorenstein liaison theory methods, we establish the following key result, which provides a simple recursive formula for the Hilbert series.

Lemma 5.3. Let $\lambda=\left(\lambda_{1}, \ldots, \lambda_{n}\right) \in \mathbb{N}^{n}$ be a partition with $\lambda_{n} \geq 2$. Set $\lambda^{\prime \prime}=$ $\left(\lambda_{1}, \ldots, \lambda_{n-1}, \lambda_{n}-1\right) \in \mathbb{N}^{n}$ and $\lambda^{\prime}=\left(\lambda_{1}-\lambda_{n}+1, \ldots, \lambda_{n-1}-\lambda_{n}+1\right) \in \mathbb{N}^{n-1}$. If $n \geq 3$, then there is the following relation among Hilbert series:

$$
p_{\lambda}(t)=p_{\lambda^{\prime \prime}}(t)+t \cdot p_{\lambda^{\prime}}(t) .
$$

Proof. We need some more notation. Given the partition $\lambda \in \mathbb{Z}^{n}$, we define $S:=$ $K[\mathbf{T}]$ as the polynomial ring in the $\lambda_{1}+\ldots+\lambda_{n}$ variables $T_{i j}$ and the ideal $J_{\lambda} \subset S$ by $S / J_{\lambda}:=K\left[G_{\lambda}\right]$. Let $\mathbf{T}^{\prime \prime}$ be the Ferrers tableau associated to $\lambda^{\prime \prime}$ and let $\widetilde{\mathbf{T}}$ be the Ferrers tableau for the partition $\widetilde{\lambda}:=\left(\lambda_{1}, \ldots, \lambda_{n-1}\right) \in \mathbb{Z}^{n-1}$. Furthermore, denote by $N$ and $\widetilde{\widetilde{T}}$ the subtableaux of $\widetilde{\mathbf{T}}$ consisting of the first $\left(\lambda_{n}-1\right)$ and the remaining columns, respectively. Let $I_{1}(N)$ be the ideal generated by the entries of $N$ and let $I_{2}(\widetilde{\mathbf{T}})$ be the ideal generated by the $2 \times 2$ minors whose entries are in $\widetilde{\widetilde{\mathbf{T}}}$. Finally, let $V, W, V^{\prime} \subset \operatorname{Proj}(S)$ be the subvarieties that are defined by $J_{\lambda}, J_{\lambda^{\prime \prime}}$, and $J_{\tilde{\lambda}}+I_{1}(N)$, respectively.

In [24, proof of Theorem 2.1], Gorla shows that $V$ is an elementary biliaison of $V^{\prime}$ on $W$. Thus, $V$ is linearly equivalent to a basic double link of $V^{\prime}$ on $W$. In particular, both have the same Hilbert function. If follows (see, for instance, 33, Lemma 4.8]) that the Hilbert functions satisfy for all integers $j$ :

$$
h_{V}(j)=h_{V^{\prime}}(j-1)+h_{W}(j)-h_{W}(j-1) .
$$

In terms of Hilbert series this reads as

$$
P\left(S / J_{\lambda}, t\right)=t \cdot P\left(S / I_{V^{\prime}}, t\right)+(1-t) \cdot P\left(S / J_{\lambda^{\prime \prime}} S, t\right) .
$$

Thus we get using Proposition 5.1 .

$$
\frac{p_{\lambda}(t)}{(1-t)^{m+n-1}}=t \cdot P\left(S / I_{V^{\prime}}, t\right)+(1-t) \cdot \frac{p_{\lambda^{\prime \prime}}(t)}{(1-t)^{m+n}}
$$

because $P\left(S / J_{\lambda^{\prime \prime}} S, t\right)=\frac{1}{1-t} \cdot P\left(K\left[G_{\lambda^{\prime \prime}}\right], t\right)=\frac{p_{\lambda^{\prime \prime}}(t)}{(1-t)^{m+n}}$. 
The definition of the homogeneous ideal of $V^{\prime}$ implies

$$
I_{V^{\prime}}=I_{2}(\widetilde{\widetilde{T}})+I_{1}(N)
$$

It follows that $S / I_{V^{\prime}}$ is isomorphic to a polynomial ring in $\lambda_{n}$ variables over $K[\widetilde{\widetilde{\mathbf{T}}}] / I_{2}(\widetilde{\widetilde{\mathbf{T}}}) \cong K\left[G_{\lambda^{\prime}}\right]$. Since $\operatorname{dim} K\left[G_{\lambda^{\prime}}\right]=m+n-\lambda_{n}-1$, we get

$$
\begin{aligned}
P\left(S / I_{V^{\prime}}, t\right) & =\frac{1}{(1-t)^{\lambda_{n}}} \cdot P\left(K\left[G_{\lambda^{\prime}}\right], t\right)=\frac{1}{(1-t)^{\lambda_{n}}} \cdot \frac{p_{\lambda^{\prime}}(t)}{(1-t)^{m+n-\lambda_{n}-1}} \\
& =\frac{p_{\lambda^{\prime}}(t)}{(1-t)^{m+n-1}} .
\end{aligned}
$$

Substituting in Equation (5.1), the claim follows.

As a first consequence, we derive an explicit formula for the Hilbert series. Observe that all terms are non-negative.

Theorem 5.4. Let $\lambda=\left(\lambda_{1}, \ldots, \lambda_{n}\right) \in \mathbb{N}^{n}$ be a partition with $n \geq 2$. Then the numerator of the normalized Hilbert series of $K\left[G_{\lambda}\right]$ is

$$
p_{\lambda}(t)=1+h_{1}(\lambda) \cdot t+\cdots+h_{n-1}(\lambda) \cdot t^{n-1},
$$

where

$$
h_{1}(\lambda)=\sum_{j=2}^{n}\left(\lambda_{j}-1\right)
$$

and

$$
h_{k}(\lambda)=\sum_{2 \leq i_{1}<i_{2}<\ldots<i_{k} \leq n} \sum_{j_{k-1}=\lambda_{i_{1}}-\lambda_{i_{k}}-k+2}^{\lambda_{i_{1}}-k} \sum_{j_{k-2}=\lambda_{i_{1}}-\lambda_{i_{k-1}}-k+3}^{j_{k-1}} \ldots \sum_{j_{1}=\lambda_{i_{1}}-\lambda_{i_{2}}}^{j_{2}} j_{1},
$$

for $k \geq 2$.

Proof. We use the notation introduced in Lemma 5.3 and its proof. This result implies for all $k \in \mathbb{N}$ :

$$
h_{k}(\lambda)=h_{k}\left(\lambda^{\prime \prime}\right)+h_{k-1}\left(\lambda^{\prime}\right) .
$$

It is easy to see that this recursion provides the formula for $h_{1}(\lambda)$.

We now induct on $n \geq 2$. If $n=2$, the minimal free resolution of $\mathcal{F}\left(I_{\lambda}\right)$ is given by an Eagon-Northcott complex. This implies in particular that

$$
p_{\lambda}(t)=1+\left(\lambda_{2}-1\right) \cdot t,
$$

as claimed. Let $n \geq 3$. Now we induct on $k \geq 2$. Since the case $k=2$ is similar, but easier than the general case, we assume $k \geq 3$. We now induct on $\lambda_{n} \geq 1$. If $\lambda_{n}=1$, then the sum $\sum_{j_{k-1}=\lambda_{i_{1}}-\lambda_{n}-k+2}^{\lambda_{i_{1}}-k}$ vanishes. Thus in the formula for $h_{k}(\lambda)$ all sums with $i_{k}=n$ vanish. This implies that we have to show $h_{k}(\lambda)=h_{k}(\widetilde{\lambda})$, where $\widetilde{\lambda}=\left(\lambda_{1}, \ldots, \lambda_{n-1}\right)$. But this is true because the ideals $J_{\lambda}$ and $J_{\widetilde{\lambda}}$ have the same generators. 
Finally, we may assume that $\lambda_{n} \geq 2$. Then the induction hypotheses and Formula (5.2) provide by distinguishing the cases $i_{k}<n$ and $i_{k}=n$ :

$$
\begin{aligned}
& h_{k}(\lambda) \\
& =\sum_{2 \leq i_{1}<\ldots<i_{k} \leq n-1} \sum_{j_{k-1}=\lambda_{i_{1}}-\lambda_{i_{k}}-k+2}^{\lambda_{i_{1}}-k} \sum_{j_{k-2}=\lambda_{i_{1}}-\lambda_{i_{k-1}}-k+3}^{j_{k-1}} \ldots \sum_{j_{1}=\lambda_{i_{1}}-\lambda_{i_{2}}}^{j_{2}} j_{1} \\
& +\sum_{2 \leq i_{1}<\ldots<i_{k-1} \leq n-1} \sum_{j_{k-1}=\lambda_{i_{1}}-\lambda_{n}-k+3}^{\lambda_{i_{1}}-k} \sum_{j_{k-2}=\lambda_{i_{1}}-\lambda_{i_{k-1}}-k+3}^{j_{k-1}} \ldots \sum_{j_{1}=\lambda_{i_{1}}-\lambda_{i_{2}}}^{j_{2}} j_{1} \\
& +\sum_{2 \leq i_{1}<\ldots<i_{k-1} \leq n-1} \sum_{j_{k-2}=\lambda_{i_{1}}-\lambda_{i_{k-1}}-k+3}^{\lambda_{i_{1}}-\lambda_{n}-k+2} \sum_{j_{k-3}=\lambda_{i_{1}}-\lambda_{i_{k-2}}-k+4}^{j_{k-2}} \ldots \sum_{j_{1}=\lambda_{i_{1}}-\lambda_{i_{2}}}^{j_{2}} j_{1} \\
& =\sum_{2 \leq i_{1}<\ldots<i_{k} \leq n-1} \sum_{j_{k-1}=\lambda_{i_{1}}-\lambda_{i_{k}}-k+2}^{\lambda_{i_{1}}-k} \sum_{j_{k-2}=\lambda_{i_{1}}-\lambda_{i_{k-1}}-k+3}^{j_{k-1}} \ldots \sum_{j_{1}=\lambda_{i_{1}}-\lambda_{i_{2}}}^{j_{2}} j_{1} \\
& +\sum_{2 \leq i_{1}<\ldots<i_{k-1} \leq n-1} \sum_{j_{k-1}=\lambda_{i_{1}}-\lambda_{n}-k+2}^{\lambda_{i_{1}}-k} \sum_{j_{k-2}=\lambda_{i_{1}}-\lambda_{i_{k-1}}-k+3}^{j_{k-1}} \ldots \sum_{j_{1}=\lambda_{i_{1}}-\lambda_{i_{2}}}^{j_{2}} j_{1} \\
& =\sum_{2 \leq i_{1}<i_{2} \ldots<i_{k} \leq n} \sum_{j_{k-1}=\lambda_{i_{1}}-\lambda_{i_{k}}-k+2}^{\lambda_{i_{1}}-k} \sum_{j_{k-2}=\lambda_{i_{1}}-\lambda_{i_{k-1}}-k+3}^{j_{k-1}} \ldots \sum_{j_{1}=\lambda_{i_{1}}-\lambda_{i_{2}}}^{j_{2}} j_{1} .
\end{aligned}
$$

This completes the proof.

Remark 5.5. It is well known that the coefficient $h_{i}(\lambda)$ of $t^{i}$ in $p_{\lambda}(t)$ has a combinatorial interpretation. In fact, interpreting the Ferrers tableau as a bounded region in the lattice $\mathbb{Z}^{2}, h_{i}(\lambda)$ is the number of lattice paths inside $\mathbf{T}_{\lambda}$ that start in the southwest corner, end in the northeast corner, and have exactly $i$ east-north turns (see [1, 22, 32, 36]).

For the multiplicity of $K\left[G_{\lambda}\right]$ we obtain a somewhat simpler formula:

\section{Corollary 5.6.}

$$
e\left(K\left[G_{\lambda}\right]\right)=\sum_{j_{n-2}=\lambda_{2}-\lambda_{n}+1}^{\lambda_{2}} \sum_{j_{n-3}=\lambda_{2}-\lambda_{n-1}+1}^{j_{n-2}} \ldots \sum_{j_{1}=\lambda_{2}-\lambda_{3}+1}^{j_{2}} j_{1} .
$$

Proof. Since $e\left(\mathcal{F}\left(I_{\lambda}\right)\right)=p_{\lambda}(1)$, this follows from Theorem 5.4. However, computationally, it is easier to use more directly Lemma 5.3 which implies $e\left(K\left[G_{\lambda}\right]\right)=$ $e\left(K\left[G_{\lambda^{\prime}}\right]\right)+e\left(K\left[G_{\lambda^{\prime \prime}}\right]\right)$.

The method of proof, using Gorenstein liaison theory, applies to all ladder determinantal ideals 24. However, here we restrict ourselves to the ideals related to Ferrers graphs, i.e. to one-sided ladder determinantal ideals generated by $2 \times 2$ minors.

We recall that for a finitely generated graded module $M$ (over an affine $K$ algebra) a suitable measure for the complexity of its resolution (hence of $M$ itself) is given by the Castelnuovo-Mumford regularity $\operatorname{reg}(M)$, that is, $\max \left\{j-i \mid \beta_{i j} \neq 0\right\}$, where $\beta_{i j}$ are the graded Betti numbers of $M$. On the other hand, the $a$-invariant 
$a(M)$ of $M$ is the degree of the Hilbert series of $M$ as a rational function. In general these numbers are related by $a(M) \leq \operatorname{reg}(M)-\operatorname{depth}(M)$, with equality if $M$ is Cohen-Macaulay. In the latter case, we thus have that $\operatorname{reg}(M)$ equals the degree of the numerator $p_{M}(t)$ of the Hilbert series of $M$. One can also interpret $a(M)$ in terms of non-vanishing of the top local cohomology of $M$. The approach we followed thus far allows us to easily compute the Castelnuovo-Mumford regularity and the $a$-invariant of the toric ring $K\left[G_{\lambda}\right]$. Before stating our results, we recall that for a partition $\lambda$, we set $s:=s(\lambda):=\lambda_{2}^{*}$. Note that $\lambda_{s} \geq 2$.

Proposition 5.7. Let $\lambda$ be a partition such that $\lambda_{2} \geq 2$. Then the CastelnuovoMumford regularity of the toric ring of the Ferrers graph $G_{\lambda}$ is

$$
\begin{aligned}
\operatorname{reg}\left(K\left[G_{\lambda}\right]\right) & =\min \left\{\lambda_{2}^{*}-1,\left\{\lambda_{j}+j-3 \mid 2 \leq j \leq \lambda_{2}^{*}=: s\right\}\right\} \\
& = \begin{cases}s-1 & \text { if } \lambda_{s} \geq 3 \\
\min \left\{j-1 \mid \lambda_{j}=2\right\} & \text { if } \lambda_{s}=2 .\end{cases}
\end{aligned}
$$

Proof. The second equality follows simply by evaluating the minimum using $\lambda_{s}+$ $s-3>s-1$ if $\lambda_{s} \geq 3$. In order to show the first equality, we note that the Cohen-Macaulayness of $K\left[G_{\lambda}\right]$ implies that $\operatorname{reg}\left(K\left[G_{\lambda}\right]\right)=\operatorname{deg} p_{\lambda}(t)$. Now for this proof denote by $r_{\lambda}-1$ the right-hand side of the claim. Then we have to show that $\operatorname{deg} p_{\lambda}=r_{\lambda}-1$. This follows directly from Theorem 5.4. Alternatively, we can use Lemma 5.3, and it suffices to show (using its notation):

$$
r_{\lambda}=\max \left\{r_{\lambda^{\prime \prime}}, 1+r_{\lambda^{\prime}}\right\} .
$$

But this can be easily checked.

Corollary 5.8. Let $\lambda$ be a partition such that $\lambda_{2} \geq 2$. Then the a-invariant of the toric ring of the Ferrers graph $G_{\lambda}$ is

$$
a\left(K\left[G_{\lambda}\right]\right)=-(n+m-1)+\min \left\{\lambda_{2}^{*}-1,\left\{\lambda_{j}+j-3 \mid 2 \leq j \leq \lambda_{2}^{*}\right\}\right\} .
$$

Proof. This result follows by applying Theorem [5.1 the equality $a\left(K\left[G_{\lambda}\right]\right)=$ $-\operatorname{dim}\left(K\left[G_{\lambda}\right]\right)+\operatorname{reg}\left(K\left[G_{\lambda}\right]\right)$ and Proposition 5.7.

Remark 5.9. We find it noteworthy, at this stage, to highlight an existing connection between $a$-invariants and Integer Programming techniques, as pointed out by Valencia and Villarreal in 49. In fact, for the type of ideals considered in this paper, the computation of the $a$-invariant amounts to the computation of the maximum number of edge disjoint directed cuts or equivalently to the minimum cardinality of the edge set that contains at least one edge of each directed cut. The latter is not easily computable using combinatorial techniques; thus it is remarkable that Corollary 5.8 provides an explicit formula.

We conclude this section by discussing particular classes of Ferrers graphs where the formulas simplify considerably. In Example 5.10 we recover in a simple way the expression for the multiplicity (due to Herzog and Trung [32) and the coefficients of the Hilbert series of the $2 \times 2$ minors of a generic $n \times m$ matrix (due to Conca and Herzog [12]). In the same simple fashion, we recover in Example 5.11] a result that appears in [53.

Example 5.10. Let $2 \leq n, m$ be integers and consider the partition $\lambda=\left(\lambda_{1}, \ldots, \lambda_{n}\right)$, where $\lambda_{i}:=m$; i.e. $G_{\lambda}$ is the complete bipartite graph $\mathcal{K}_{n, m}$. Then the coefficients of 
the polynomial in the Hilbert series of the toric ring $K\left[G_{\lambda}\right]$ as well as its multiplicity are

$$
h_{k}(\lambda)=\left(\begin{array}{c}
m-1 \\
k
\end{array}\right)\left(\begin{array}{c}
n-1 \\
k
\end{array}\right) \quad \text { and } \quad e\left(K\left[G_{\lambda}\right]\right)=\left(\begin{array}{c}
n+m-2 \\
m-1
\end{array}\right) .
$$

Proof. In the calculations that will follow we will make repeated use of the combinatorial identity

$$
\sum_{j_{t}=1}^{j_{t+1}}\left(\begin{array}{c}
j_{t}+t-1 \\
j_{t}-1
\end{array}\right)=\left(\begin{array}{c}
j_{t+1}+(t+1)-1 \\
j_{t+1}-1
\end{array}\right)
$$

which can be found in [25. Formula 1.49]. According to Theorem 5.4 we only need to show the formula for $h_{k}$ when $k \geq 2$, as in the other two cases the expression is trivially verified. In this particular case the expression in Theorem 5.4 reduces to

$$
\begin{aligned}
& h_{k}(\lambda)=\sum_{2 \leq i_{1}<i_{2}<\ldots<i_{k} \leq n} \sum_{j_{k-1}=-k+2}^{m-k} \sum_{j_{k-2}=-k+3}^{j_{k-1}} \ldots \sum_{j_{2}=-1}^{j_{3}} \sum_{j_{1}=0}^{j_{2}} j_{1} \\
& =\sum_{2 \leq i_{1}<i_{2}<\ldots<i_{k} \leq n} \sum_{j_{k-1}=-k+2}^{m-k} \sum_{j_{k-2}=-k+3}^{j_{k-1}} \ldots \sum_{j_{2}=-1}^{j_{3}}\left(\begin{array}{c}
j_{2}+1 \\
2
\end{array}\right) \\
& =\sum_{2 \leq i_{1}<i_{2}<\ldots<i_{k} \leq n} \sum_{j_{k-1}=-k+2}^{m-k} \sum_{j_{k-2}=-k+3}^{j_{k-1}} \ldots \sum_{j_{3}=-2}^{j_{4}}\left(\begin{array}{c}
j_{3}+2 \\
3
\end{array}\right) \\
& =\sum_{2 \leq i_{1}<i_{2}<\ldots<i_{k} \leq n} \sum_{j_{k-1}=-k+2}^{m-k}\left(\begin{array}{c}
j_{k-1}+(k-1)-1 \\
j_{k-1}-1
\end{array}\right) \\
& =\sum_{2 \leq i_{1}<i_{2}<\ldots<i_{k} \leq n}\left(\begin{array}{c}
m-1 \\
k
\end{array}\right)=\left(\begin{array}{c}
m-1 \\
k
\end{array}\right)\left(\begin{array}{c}
n-1 \\
k
\end{array}\right) \text {. }
\end{aligned}
$$

Finally, the expression for the multiplicity of $K\left[G_{\lambda}\right]$ follows from Corollary [5.6 by performing similar computations.

Example 5.11. Let $2 \leq n \leq m$ be integers and consider the partition $\lambda=$ $\left(\lambda_{1}, \ldots, \lambda_{n}\right)$ where $\lambda_{k}:=m+1-k$. Then the Hilbert series of the toric ring $K\left[G_{\lambda}\right]$ is

$$
P\left(K\left[G_{\lambda}\right], t\right)=\frac{1+h_{1}(\lambda) \cdot t+\cdots+h_{n-1}(\lambda) \cdot t^{n-1}}{(1-t)^{m+n-1}},
$$

where

$$
h_{k}(\lambda)=\left(\begin{array}{c}
n-1 \\
k
\end{array}\right)\left(\begin{array}{c}
m-2 \\
k
\end{array}\right)-\left(\begin{array}{l}
n-1 \\
k+1
\end{array}\right)\left(\begin{array}{c}
m-2 \\
k-1
\end{array}\right)
$$

for all $k=0, \ldots, n-1$. In particular, the multiplicity is:

$$
e\left(K\left[G_{\lambda}\right]\right)=\frac{m-n+1}{m} \cdot\left(\begin{array}{c}
m+n-2 \\
m-1
\end{array}\right) .
$$

Proof. We induct on $n \geq 2$. Theorem 5.4 immediately provides the claim about $h_{0}(\lambda)$ and $h_{1}(\lambda)$. Thus we may assume $n \geq 3$. Consider the partition $\bar{\lambda}=$ $(m, \ldots, m+2-n, j)$ where $1 \leq j \leq m+1-n$; i.e. $\bar{\lambda}$ differs from the given partition $\lambda$ at most in the last entry. Then, using the notation of Lemma 5.3 if $j \geq 2$, we get $\overline{\lambda^{\prime \prime}}=(m, \ldots, m+2-n, j-1) \in \mathbb{N}^{n}$ and $\overline{\lambda^{\prime}}=(m-j+1, \ldots, m-j+3-n) \in \mathbb{N}^{n-1}$. 
Note that the induction hypothesis applies to $\bar{\lambda}^{\prime}$. Letting $j$ vary between 1 and $m+1-n$, Lemma 5.3 and the induction hypothesis provide

$$
\begin{aligned}
h_{k}(\lambda)= & h_{k}(m, m-1, \ldots, m+2-n) \\
& +\sum_{j=2}^{m+1-n} h_{k-1}(m-j+1, \ldots, m-j+3-n) \\
= & \left(\begin{array}{c}
n-2 \\
k
\end{array}\right)\left(\begin{array}{c}
m-2 \\
k
\end{array}\right)-\left(\begin{array}{c}
n-2 \\
k+1
\end{array}\right)\left(\begin{array}{c}
m-2 \\
k-1
\end{array}\right) \\
& +\sum_{j=2}^{m+1-n}\left[\left(\begin{array}{c}
n-2 \\
k-1
\end{array}\right)\left(\begin{array}{c}
m-j-1 \\
k-1
\end{array}\right)-\left(\begin{array}{c}
n-2 \\
k
\end{array}\right)\left(\begin{array}{c}
m-j-1 \\
k-2
\end{array}\right)\right] \\
= & \left(\begin{array}{c}
n-2 \\
k
\end{array}\right)\left(\begin{array}{c}
m-2 \\
k
\end{array}\right)-\left(\begin{array}{c}
n-2 \\
k+1
\end{array}\right)\left(\begin{array}{c}
m-2 \\
k-1
\end{array}\right) \\
& +\left(\begin{array}{c}
n-2 \\
k-1
\end{array}\right) \cdot\left[\left(\begin{array}{c}
m-2 \\
k
\end{array}\right)-\left(\begin{array}{c}
n-2 \\
k
\end{array}\right)\right]-\left(\begin{array}{c}
n-2 \\
k
\end{array}\right) \cdot\left[\left(\begin{array}{c}
m-2 \\
k-1
\end{array}\right)-\left(\begin{array}{c}
n-2 \\
k-1
\end{array}\right)\right] \\
= & \left(\begin{array}{c}
n-1 \\
k
\end{array}\right)\left(\begin{array}{c}
m-2 \\
k
\end{array}\right)-\left(\begin{array}{c}
n-1 \\
k+1
\end{array}\right)\left(\begin{array}{c}
m-2 \\
k-1
\end{array}\right),
\end{aligned}
$$

as claimed. Finally, as noted earlier, $e\left(K\left[G_{\lambda}\right]\right)=p_{\lambda}(1)$. Thus, using [25, Formula 3.20 , we get

$$
\begin{aligned}
e\left(K\left[G_{\lambda}\right]\right) & =\sum_{k=0}^{n-1}\left[\left(\begin{array}{c}
n-1 \\
k
\end{array}\right)\left(\begin{array}{c}
m-2 \\
k
\end{array}\right)-\left(\begin{array}{l}
n-1 \\
k+1
\end{array}\right)\left(\begin{array}{c}
m-2 \\
k-1
\end{array}\right)\right] \\
& =\left(\begin{array}{c}
m+n-3 \\
n-1
\end{array}\right)-\left(\begin{array}{c}
m+n-3 \\
n-3
\end{array}\right) \\
& =\frac{m-n+1}{m} \cdot\left(\begin{array}{c}
m+n-2 \\
m-1
\end{array}\right),
\end{aligned}
$$

where the last equality is easy to verify.

As reflected in the coefficients $h_{k}$, one should observe that the roles of $m$ and $n$ are not symmetric in the previous corollary, since we consider the partition $\lambda=$ $(m, m-1, \ldots, m-n+1)$. However, in the case $m=n$, the above formulæ greatly simplify (becoming symmetric!) and the rings have particularly good properties:

Example 5.12. Consider the partition $\lambda=(n, n-1, \ldots, 2,1) \in \mathbb{Z}^{n}$. The ring $R / I_{\lambda}$ cogenerated by the edge ideal $I_{\lambda}$ of the associated Ferrers graph $G_{\lambda}$ is CohenMacaulay (by Corollary 2.8) with multiplicity $n+1$ and Hilbert series

$$
P\left(R / I_{\lambda}, t\right)=\frac{1+n t}{(1-t)^{n}} .
$$

The toric ring $K\left[G_{\lambda}\right]$ is Gorenstein with Hilbert series:

$$
P\left(K\left[G_{\lambda}\right], t\right)=\frac{\sum_{k=0}^{n-2} \frac{\left(\begin{array}{c}
n-2 \\
k
\end{array}\right)\left(\begin{array}{c}
n-1 \\
k
\end{array}\right)}{k+1} t^{k}}{(1-t)^{2 n-1}} .
$$


In particular, the multiplicity of $K\left[G_{\lambda}\right]$ is the Catalan number:

$$
e\left(K\left[G_{\lambda}\right]\right)=\frac{\left(\begin{array}{c}
2(n-1) \\
n-1
\end{array}\right)}{n} .
$$

We refer the interested reader to [47, 48, for a wealth of information about Catalan numbers.

\section{ACKNOWLEDGEMENT}

The authors would like to thank the anonymous referee for the careful reading of the original manuscript and helpful comments.

\section{REFERENCES}

[1] S.S. Abhyankar, Enumerative combinatorics of Young tableaux, Marcel Dekker, New York, Basel, 1988. MR926272 (89e:05011)

[2] F. Brenti, G. Royle and D. Wagner, Location of zeros of chromatic and related polynomials of graphs, Canad. J. Math. 46 (1994), 55-80. MR.1260339 (94k:05077)

[3] D. Bayer and B. Sturmfels, Cellular resolutions of monomial modules, J. Reine Angew. Math. 502 (1998), 123-140. MR 1647559 (99g:13018)

[4] D. Bayer, I. Peeva and B. Sturmfels, Monomial resolutions, Math. Res. Lett. 5 (1998), 31-46. MR:1618363 (99c:13029)

[5] R. Biagioli, S. Faridi and M. Rosas, Resolutions of De Concini-Procesi ideals of hooks, Communications in Algebra 35 (2007), 3875-3891. MR 2371263

[6] W. Bruns and A. Guerrieri, The Dedekind-Mertens formula and determinantal rings, Proc. Amer. Math. Soc. 127 (1999), 657-663. MR1468185 (99f:13013)

[7] W. Bruns and J. Herzog, On the computation of a-invariants, Manuscripta Math. 77 (1992), 201-213. MR1188581 (93k:13032)

[8] W. Bruns and J. Herzog, Cohen-Macaulay rings, Cambridge Studies in Advanced Mathematics 39, Cambridge University Press, Cambridge, 1993. MR.1251956 (95h:13020)

[9] F. Butler, Rook theory and cycle-counting permutation statistics, Adv. in Appl. Math. 33 (2004), 655-675. MR2095860 (2005j:05002)

[10] A. Conca, Ladder determinantal rings, J. Pure Appl. Algebra 98 (1995), 119-134. MR.1319965 (96a:13013)

[11] A. Conca, Straightening law and powers of determinantal ideals of Hankel matrices, Adv. Math. 138 (1998), 263-292. MR.1645574 (99i:13020)

[12] A. Conca and J. Herzog, On the Hilbert function of determinantal rings and their canonical module, Proc. Amer. Math. Soc. 112 (1994), 677-681. MR.1213858(95a:13016)

[13] A. Corso and U. Nagel, Specializations of Ferrers ideals, to appear in J. Algebraic Combin.

[14] A. Corso, W.V. Vasconcelos and R.H. Villarreal, Generic Gaussian ideals, J. Pure Appl. Algebra 125 (1998), 117-127. MR:1600012 (98m:13014)

[15] M. Develin, Rook poset equivalence of Ferrers boards, Order 23 (2006), 179-195. MR 2308905

[16] K. Ding, Rook placements and cellular decomposition of partition varieties, Discrete Mathematics 170 (1997), 107-151. MR 1452940 (98i:05166)

[17] J.A. Eagon and D.G. Northcott, Ideals defined by matrices and a certain complex associated with them, Proc. Roy. Soc. Ser. A 269 (1962), 188-204. MR0142592 (26:161)

[18] R. Ehrenborg and S. van Willigenburg, Enumerative properties of Ferrers graphs, Discrete Comput. Geom. 32 (2004), 481-492. MR2096744 (2005j:05076)

[19] D. Eisenbud, M. Green, K. Hulek and S. Popescu, Restricting linear syzygies: Algebra and geometry, Compositio Math. 141 (2005), 1460-1478. MR2188445 (2006m:14072)

[20] D. Eisenbud, M. Green, K. Hulek and S. Popescu, Small schemes and varieties of minimal degree, Amer. J. Math. 128 (2006), 1363-1389. MR2275024 (2007j:14078)

[21] R. Fröberg, On Stanley-Reisner rings, in Topics in algebra, Part 2 (Warsaw, 1988), pp. 57-70, Banach Center Publ. 26, PWN, Warsaw, 1990. MR.1171260 (93f:13009)

[22] I. Gessel and G. Viennot, Binomial determinants, paths, and hook length formulae, Adv. Math. 58 (1985), 300-321. MR815360 (87e:05008) 
[23] J. Goldman, J.T. Joichi and D. White, Rook Theory I. Rook equivalence of Ferrers boards, Proc. Amer. Math. Soc. 52 (1975), 485-492. MR0429578 (55:2590)

[24] E. Gorla, Mixed ladder determinantal varieties from two-sided ladders, J. Pure Appl. Algebra 211, (2007) 433-444. MR.2340461

[25] H.W. Gould, Combinatorial identities: A standardized set of tables listing 500 binomial coefficient summations (rev. ed.), Morgantown, West Virginia, 1972. MR0354401(50:6879)

[26] S.R. Ghorpade, Hilbert functions of ladder determinantal varieties, Discrete Math. 246 (2002), 131-175. MR:1887484 (2003e:13026)

[27] D. Gouyou-Beauchamps, Chemins sous-diagonaux et tableau de Young, in Combinatoire Enumerative (Montreal, 1985), pp. 112-125, Lect. Notes Math. 1234, 1986. MR 927762 (89d:05005)

[28] H.T. Hà and A. Van Tuyl, Splittable ideals and the resolutions of monomial ideals, J. Algebra 309 (2007), 405-425. MR 2301246 (2008a:13016)

[29] J. Haglund, Rook theory and hypergeometric series, Adv. in Appl. Math. 17 (1996), 408-459. MR:1422066 (98k:33010)

[30] J. Herzog and T. Hibi, Distributive lattices, bipartite graphs and Alexander duality, J. Algebraic Combin. 22 (2005), 289-302. MR2181367(2006h:06004)

[31] J. Herzog, T. Hibi and X. Zheng, Monomial ideals whose powers have a linear resolution, Math. Scand. 95 (2004), 23-32. MR2091479 (2005f:13012)

[32] J. Herzog and N.V. Trung, Gröbner bases and multiplicity of determinantal and Pfaffian ideals, Adv. Math. 96 (1992), 1-37. MR1185786 (94a:13012)

[33] J. Kleppe, J. Migliore, R.M. Miró-Roig, U. Nagel and C. Peterson, Gorenstein liaison, complete intersection liaison invariants and unobstructedness, Mem. Amer. Math. Soc. 154 (2001), no. 732. MR1848976 (2002e:14083)

[34] C. Krattenthaler, Non-crossing two-rowed arrays and summations for Schur functions, preprint 1992.

[35] C. Krattenthaler and S.G. Mohanty, On lattice path counting by major index and descents, Europ. J. Combin. 14 (1993), 43-51. MR.1197475 (94e:05019)

[36] C. Krattenthaler and M. Prohaska, A remarkable formula for counting non-intersecting lattice paths in a ladder with respect to turns, Trans. Amer. Math. Soc. 351 (1999), 1015-1042. MR.1407495 (99e:05009)

[37] C. Krattenthaler and M. Rubey, A determinantal formula for the Hilbert series of one-sided ladder determinantal rings, in Algebra, arithmetic and geometry with applications (West Lafayette, IN, 2000), pp. 525-551, Springer, Berlin, 2004. MR2037108(2005a:13026)

[38] D.M. Kulkarni, Counting of paths and coefficients of the Hilbert polynomial of a determinantal ideal, Discrete Math. 154 (1996), 141-151. MR.1395454 (97f:13018)

[39] J. Migliore, Introduction to liaison theory and deficiency modules, Progress in Mathematics 165, Birkhäuser, 1998. MR1712469 (2000g:14058)

[40] J. Migliore, U. Nagel and T. Römer, Extensions of the multiplicity conjecture, to appear in Trans. Amer. Math. Soc.

[41] E. Miller and B. Sturmfels, Combinatorial commutative algebra, Graduate Texts in Mathematics 227, Springer-Verlag, New York, 2005. MR 2110098 (2006d:13001)

[42] A. Mitchell, The inverse rook problem on Ferrers boards, preprint 2004.

[43] H. Narasimhan, The irreducibility of ladder determinantal varieties, J. Algebra 102 (1986), 162-185. MR853237 (87j:14081)

[44] C. Polini, B. Ulrich and M. Vitulli, The core of zero-dimensional monomial ideals, Adv. Math. 211 (2007), 72-93. MR2313528(2008b:13033)

[45] M. Rubey, The h-vector of a ladder determinantal ring cogenerated by $2 \times 2$ minors is logconcave, J. Algebra 292 (2005), 303-323. MR2172157(2007a:05147)

[46] A. Simis, W.V. Vasconcelos and R. Villarreal, On the ideal theory of graphs, J. Algebra 167 (1994), 389-416. MR 1283294 (95e:13002)

[47] R.P. Stanley, Enumerative combinatorics, Vol. 2, Cambridge Studies in Advanced Mathematics 62, Cambridge University Press, Cambridge, 1999. MR.1676282 (2000k:05026)

[48] R.P. Stanley, Catalan addendum (URL: www-math.mit.edu/ $\sim$ rstan/ec/catadd.pdf).

[49] C. Valencia and R. Villarreal, Canonical modules of certain edge subrings, European J. Combin. 24 (2003), 471-487. MR1983672(2004c:13033)

[50] A. Varvak, Rook numbers and the normal ordering problem, J. Combin. Theory Ser. A 112 (2005), 292-307. MR2177488 (2006h:05011) 
[51] R. Villarreal, Monomial algebras, Monographs and Textbooks in Pure and Applied Mathematics 238, Marcel Dekker, Inc., New York, 2001. MR1800904 (2002c:13001)

[52] H.-J. Wang, A determinantal formula for the Hilbert series of determinantal rings of onesided ladder, J. Algebra 265 (2003), 79-99. MR1984900 (2004d:13016)

[53] H.-J. Wang, Counting of paths and the multiplicity of determinantal rings, preprint 2002.

[54] H.-J. Wang, A conjecture of Herzog and Conca on counting of paths, preprint 2002.

Department of Mathematics, University of Kentucky, Lexington, Kentucky 40506

E-mail address: corso@ms.uky.edu

$U R L$ : www.ms.uky.edu/ ${ }^{\sim} \operatorname{corso}$

Department of Mathematics, University of Kentucky, Lexington, Kentucky 40506

E-mail address: uwenagel@ms.uky.edu

$U R L$ : www.ms . uky.edu/ uwenagel 